\title{
Individuelle Nutzung unterrichtlicher Angebote - Zur Bedeutung von Lernvoraussetzungen und Unterrichtsbeteiligung
}

\author{
Nina C. Jansen (iD · Jasmin Decristan $(D) \cdot$ Benjamin Fauth $(\mathbb{D}$
}

Eingegangen: 17. Mai 2021 / Überarbeitet: 22. Dezember 2021 / Angenommen: 27. Dezember 2021 /

Online publiziert: 27. Januar 2022

(C) Der/die Autor(en) 2022

Zusammenfassung In Angebots-Nutzungs-Modellen wird die individuelle Nutzung unterrichtlicher Angebote als wichtige Voraussetzung schulischen Lernens angesehen. Nutzung wird dabei als kognitiver, wechselseitig durch motivationale und emotionale Aspekte beeinflusster Prozess innerhalb der Lernenden verstanden. Welche Bedeutung individuelle Merkmale und die Beteiligung am Unterricht für die Nutzung von Unterricht haben, ist bislang noch nicht hinreichend untersucht. Der vorliegende Beitrag widmet sich diesem Desiderat anhand von Daten aus $40 \mathrm{Ma}-$ thematikklassen $(N=932$ Schüler*innen) in der Sekundarstufe. Die Beteiligung am Unterricht wurde über Kodierungen von Unterrichtsvideos erfasst ( $N=855$ verbale Beteiligungen; $N=3850$ weitere Meldungen), individuelle Lernvoraussetzungen und die selbsteingeschätzte Intensität der Nutzung unterrichtlicher Angebote über Fragebögen und einen Vorwissenstest der teilnehmenden Schüler*innen. Die Ergebnisse zeigen Zusammenhänge sowohl zwischen individuellen kognitiven und motivationalen Merkmalen und der selbstberichteten Nutzung des Unterrichtsangebots als auch zwischen Unterrichtsbeteiligung und Nutzung. Die schüler*innengesteuerte Beteiligung leistete einen eigenständigen Beitrag zur Vorhersage von Nutzung, auch über individuelle Merkmale hinaus. Die Ergebnisse verdeutlichen die Relevanz von akti-

\footnotetext{
Nina C. Jansen $(\bowtie) \cdot$ Jasmin Decristan Institut für Bildungsforschung in der School of Education, Bergische Universität Wuppertal, Rainer-Gruenter-Straße 21, 42119 Wuppertal, Deutschland

E-Mail: njansen@uni-wuppertal.de

Jasmin Decristan

E-Mail: decristan@uni-wuppertal.de

Benjamin Fauth

Institut für Bildungsanalysen Baden-Württemberg (IBBW), Heilbronner Straße 172, 70191 Stuttgart,

Deutschland

E-Mail: benjamin.fauth@ibbw.kv.bwl.de

Universität Tübingen, Tübingen, Deutschland
} 
ver Unterrichtsbeteiligung für die Angebotsnutzung und liefern weitere Erkenntnisse im Kontext von Angebots-Nutzungs-Modellen schulischen Lernens.

Schlüsselwörter Angebots-Nutzungs-Modelle · Unterrichtsbeteiligung · Nutzungsprozesse $\cdot$ Videoanalysen

\title{
Individual use of instruction-On the relevance of students' characteristics and participation in classroom discourse
}

\begin{abstract}
In models of teaching and learning, the individual use of instruction represents an important determinant of learning. The significance of individual characteristics and participation in classroom for the use of instruction has not yet been sufficiently investigated. The present article addresses this research gap using data from 40 mathematics classes $(N=932$ students $)$ in secondary school. Participation was assessed via coding of videos $(N=855$ verbal participations; $N=3850$ further hand raisings). Individual learning characteristics and self-reported use of instruction were measured by questionnaires and a prior knowledge test. The results show correlations of cognitive and motivational characteristics with the self-reported use of instruction. Similarly, they also show the importance of participation in classroom discourses for the use of instruction. Student-driven participation made an additional contribution to the prediction of the use of instruction, even beyond individual characteristics. The results illustrate the relevance of active classroom participation for the use of instruction and provide further insight in the context of models of teaching and learning.
\end{abstract}

Keywords Models of teaching and learning - Classroom participation - Individual use of instruction - Video analyses

Unterricht wird als Interaktion lehrkraft- und schüler*innenseitiger Faktoren in einem wechselseitig ko-konstruktiven Prozess konzeptualisiert (Fend 1998). Schüler*innen nutzen dabei die unterrichtlichen Lernangebote in individuell unterschiedlicher Weise und können ihrerseits auch aktiv zur unterrichtlichen Interaktion beitragen. Welche individuellen Lernvoraussetzungen und welche unterrichtlichen Prozesse dazu führen, dass ein Unterrichtsangebot auch tatsächlich genutzt wird, ist weiterhin als Desiderat der empirischen Unterrichtsforschung zu betrachten (Vieluf et al. 2020).

\section{Individuelle Nutzung als zentrale Bedingung schulischen Lernens}

In Angebots-Nutzungs-Modellen schulischen Lernens wird die individuelle Nutzung unterrichtlicher Angebote als zentraler Mediator zwischen unterrichtlichem Angebot und Lernoutcomes angesehen (Fend 1998; Helmke 2017; Seidel 2014). Diese Relevanz des schüler*innenseitigen Umganges mit dem unterrichtlichen Angebot thematisierte Carroll (1963) bereits 25 Jahre vor der Veröffentlichung des ersten 
Angebots-Nutzungs-Modells (Fend 1998), indem er Lernzuwächse modellhaft als Funktion erhaltener Lerngelegenheiten charakterisierte. Den gewünschten Lernerfolg würden entsprechende Gelegenheiten jedoch nur hervorbringen, wenn sie durch funktionale Lernaktivitäten auch effektiv von den Lernenden genutzt würden (Carroll 1963). Demzufolge wird schüler*innenseitige Nutzung als ein korrespondierendes Element zum lehrkraftseitigen Unterrichtsangebot betrachtet (Mercer und Dawes 2014; Vygotskij 1978).

Nutzung wird in verschiedenen Angebots-Nutzungs-Modellen jedoch nicht konsistent beschrieben. Übereinstimmend wird Nutzung als komplexer und interindividuell verschiedener „kognitiver Lernprozess“ (Vieluf et al. 2020, S. 68) verstanden (Helmke 2017; Klieme et al. 2006; Kunter und Trautwein 2013; Seidel 2014) und manifestiert sich in mentalen Prozessen wie zum Beispiel intensivem Nachdenken oder dem Entwickeln von Ideen. Einige dieser Modelle (Klieme et al. 2006; Seidel 2014) ordnen dem Konstrukt der Nutzung auch motivationale und emotionale Einflüsse auf die kognitiven Prozesse zu (Vieluf et al. 2020). Vor dem Hintergrund der Komplexität innerer Lernprozesse erscheint dieser simultane Einbezug kognitiver, motivationaler und emotionaler Einflüsse als zutreffende Charakterisierung von Nutzung. Äußere Aktivitäten, wie beispielsweise die unterrichtliche Beteiligung, werden mitunter ebenso als Indikatoren für Nutzung betrachtet (Seidel 2014). Dabei sind sie jedoch als solche weniger eindeutig als Indikatoren innerer Aktivitäten interpretierbar, da sie die Koproduktion von Unterricht mitgestalten und gegebenenfalls selbst Lernangebote für andere Schüler*innen darstellen oder diese beeinflussen können (vgl. Vieluf et al. 2020, S. 70).

In ihrem Modell der Wirkungen der drei Dimensionen von Unterrichtsqualität führen Klieme und Rakoczy (2008) als Nutzungsparameter stattdessen die aktive Lernzeit, die Verarbeitungstiefe und das Erleben von Selbstbestimmung an, die wiederum den Zusammenhang von Unterrichtsqualität und schulischem Lernen mediieren. Die aktive Lernzeit (time on task) findet sich auch in den theoretischen (Carroll 1963) und empirischen Arbeiten (Hattie 2009) anderer Autor*innen als wichtiger Bestandteil des schulischen Lernens. Diesbezüglich wird angenommen, dass sich Lernende darin unterscheiden wie viel Lernzeit individuell benötigt wird, um ein Lernziel zu erreichen (Anderson 1976; Gettinger 1984). Der zweite bei Klieme und Rakoczy (2008) beschriebene Nutzungsparameter, die vertiefte kognitive Verarbeitung von Unterrichtsinhalten, fußt auf kognitionspsychologischen Theorien. Eine vertiefte Verarbeitung führt demzufolge zu einer elaborierten Speicherung und Vernetzung von Wissenselementen in bestehenden mentalen Netzwerken. In der Konsequenz können Wissensinhalte einfacher und schneller abgerufen, sowie auf andere Domänen transferiert werden (Craik und Lockhart 1972; Klauer und Leutner 2012). Entsprechend der Zuordnung zur Unterrichtsqualitätsdimension kognitive Aktivierung lässt sich die kognitive Aktiviertheit der Schüler*innen als Indikator der Verarbeitungstiefe ansehen (Pauli et al. 2008). Den dritten Nutzungsparameter bildet das Selbstbestimmungserleben (Klieme und Rakoczy 2008). Der Selbstbestimmungstheorie von Deci und Ryan (1993) zufolge lassen sich drei psychologische Grundbedürfnisse des Menschen charakterisieren: Das Erleben von Kompetenz, Autonomie und sozialer Eingebundenheit. Studien zu Folge gelten diese Grundbedürfnisse als entscheidend für die Entwicklung intrinsischer Motivation und 
die Leistung von Lernenden (Taylor et al. 2014). In der empirischen Unterrichtsforschung wird das Erleben von Selbstbestimmung vor allem mit der konstruktiven Unterstützung als Unterrichtsqualitätsdimension in Zusammenhang gebracht (Fauth et al. 2014; Rakoczy 2006).

Die vorangegangenen Darstellungen verdeutlichen, dass unterrichtliche Nutzung als innerer, mentaler Prozess definiert werden kann (Friedrich und Mandl 1992; Vieluf et al. 2020). Dies erschwert eine valide empirische Erfassung von Nutzung durch außenstehende Personen. Daher wird häufig auf Selbstberichte der Lernenden zur Operationalisierung von Nutzung zurückgegriffen (z. B. Böheim et al. 2020; Schnitzler et al. 2020), während beobachtbare äußere Aktivitäten, wie die Unterrichtsbeteiligung, Elemente des unterrichtlichen Ko-Konstruktionsprozesses bilden und sich von Nutzung abgrenzen lassen (Vieluf et al. 2020). Welche individuellen Lernvoraussetzungen und unterrichtlichen Prozesse zur Erklärung individuell variierender Nutzung herangezogen werden können, darauf wird in den folgenden Abschnitten näher eingegangen.

\section{Bedingungsfaktoren individueller Nutzung}

\subsection{Individuelle Lernvoraussetzungen}

Schüler*innen unterscheiden sich hinsichtlich soziodemografischer, kognitiver und motivational-emotionaler Merkmale in ihren Voraussetzungen für das Lernen. Entsprechend eines konstruktivistischen Verständnisses wird angenommen, dass im Zuge erfolgreichen Lernens Informationen aus Lernangeboten bewusst aufgenommen, aktiv verarbeitet und so mit den Informationen vorhandener mentaler Netzwerke verknüpft werden (Chi 2009). In Abhängigkeit von individuellen Voraussetzungen der Lernenden können diese Verarbeitungsprozesse unterschiedlich erfolgen - und somit Lernangebote unterschiedlich genutzt werden.

Auch in Angebots-Nutzungs-Modellen ist die Annahme mit modelliert, dass Lernende die bestehenden Lernangebote in Abhängigkeit von ihren individuell verschiedenen Lernvoraussetzungen unterschiedlich nutzen (Seidel 2014; Vieluf et al. 2020). Oftmals wird zwischen distalen und proximalen Lernvoraussetzungen unterschieden. Dabei gelten Geschlecht, Zuwanderungshintergrund und familiärer sozioökonomischer Status als distale Lernvoraussetzungen, während kognitive und motivationalemotionale Merkmale als proximale Lernvoraussetzungen betrachtet werden. Es wird angenommen, dass die distalen Merkmale vornehmlich indirekt, über proximale Merkmale, Einfluss auf das Lernen nehmen. Dennoch finden sich oft eigenständige Erklärungsbeiträge distaler Merkmale, wie Geschlecht, Herkunft und sozioökonomischer Status auf schulisches Lernen (Hattie 2009). Hierbei lässt sich annehmen, dass Schüler*innen abhängig von distalen, aber gleichzeitig meist gut sichtbaren Merkmalen unterschiedlich in unterrichtliche Interaktionen eingebunden sind. Mit soziodemographischen Merkmalen verknüpfte, differenzielle eigene Erfolgserwartungen und solche von Lehrkräften (Jussim und Harber 2005) können dazu führen, dass Lernende abhängig von Geschlecht sowie familiärer und kultureller Herkunft die Lernzeit weniger gut nutzen und die Inhalte weniger vertieft verarbeiten. Auch 
lassen sich Zusammenhänge mit dem Selbstbestimmungserleben, insbesondere dem Kompetenzerleben und dem Erleben sozialer Eingebundenheit erwarten.

Den stärksten Prädiktor für Lernleistungen stellen kognitive Lernvoraussetzungen dar. Erfasst werden diese oft über kognitive Grundfähigkeiten oder Vorwissen in einem Inhaltsbereich. Entsprechend kognitionspsychologischen Annahmen ermöglichen günstigere kognitive Lernvoraussetzungen eine schnellere und qualitativ bessere Verarbeitung von Lerninhalten (Chi und Wylie 2014). Umfassendes Vorwissen erleichtert die Anknüpfung erworbener Wissensinhalte an bestehende mentale Netzwerke, wodurch wiederum die weitere mentale Beschäftigung mit kontextualisierten Informationen ermöglicht wird. Eine fortgeführte Auseinandersetzung mit lernrelevanten Inhalten, zum Beispiel im Zuge aktiv genutzter Lernzeit, wird in diesem Zusammenhang reguliert und unterstützt (Schraw 2006). Ebenso sollte ein höheres Vorwissen auch mit mehr Selbstbestimmungserleben (vor allem Kompetenzerleben) einhergehen (Großmann et al. 2020).

Ebenso beeinflussen die in der pädagogisch-psychologischen Forschung prominenten Merkmale Interesse und Selbstkonzept die individuellen Lernprozesse. Im Sinne von Erwartungs-mal-Wert-Modellen (z. B. Wigfield und Eccles 2000) messen Schüler*innen mit hohem Interesse an betreffenden Inhalten diesen einen gesteigerten intrinsischen Wert bei. Eine entsprechend erhöhte intrinsische Motivation ist empirischen Befunden zufolge assoziiert mit einer vermehrten Nutzung korrespondierender Lernangebote (Taylor et al. 2014). Theoretisch lässt sich annehmen, dass entsprechende Lerninhalte häufiger, länger und elaborierter verarbeitet werden, was positive Zusammenhänge mit aktiver Lernzeit und kognitiver Aktiviertheit nahelegt (Schiefele et al. 1993a). Darüber hinaus gilt das Bestehen intrinsischer Motivation als grundlegend für das Erleben von Selbstbestimmtheit, welches dementsprechend positiv mit subjektivem Interesse assoziiert ist (Deci 1992).

Das fachspezifische Selbstkonzept basiert auf der Wahrnehmung eigener domänenspezifischer Fähigkeiten, welche bei günstiger Ausprägung, vergleichbar mit dem Interesse, zu vermehrter zeitlicher und verstärkter kognitiver Auseinandersetzung mit unterrichtlichen Inhalten führen, wie empirische Studien zeigen (Marsh und Martin 2011). Vor dem Hintergrund gesteigerten Kompetenz- und Autonomieerlebens lassen empirische Befunde außerdem einen positiven Zusammenhang des Selbstkonzeptes mit dem Selbstbestimmungserleben annehmen (Lepper et al. 2021).

Eine Vielzahl empirischer Studien untermauert die angenommenen Zusammenhänge von Vorwissen (Schraw 2006), Selbstkonzept (Böheim et al. 2020; Kriegbaum et al. 2018) und Interesse (Schiefele et al. 1993a) mit der Entwicklung schulischer Kompetenzen. Die Bedeutung der Lernvoraussetzungen für die individuelle Nutzung unterrichtlicher Angebote, im Sinne von time on task, kognitiver Aktiviertheit und dem Selbstbestimmungserleben ist dagegen bislang wenig untersucht worden.

\subsection{Unterrichtsbeteiligung als Unterrichtsprozessmerkmal}

Neben den individuellen Voraussetzungen der Lernenden wird in Angebots-Nutzungs-Modellen das unterrichtliche Angebot selbst als Bedingungsfaktor einer individuellen Nutzung angesehen (Helmke 2017; Seidel 2014; Vieluf et al. 2020). In der empirischen Unterrichtsforschung haben sich Unterrichtsqualitätsmerkmale als für 
Tab. 1 Grundmuster der Unterrichtsbeteiligung nach Sacher (1995). (Adaptiert nach Decristan et al. (2019))

\begin{tabular}{|c|c|c|c|}
\hline \multirow[b]{2}{*}{ Lehrkraft } & \multicolumn{3}{|l|}{ Schüler*in } \\
\hline & $\begin{array}{l}\text { Beteiligt sich } \\
\text { (= schüler*innenge- } \\
\text { steuerte Beteiligung) }\end{array}$ & Beteiligt sich nicht & \\
\hline $\begin{array}{l}\text { Ruft auf } \\
\text { (= lehrkraftgesteu- } \\
\text { erte Beteiligung) }\end{array}$ & $\begin{array}{l}1 \text { - Schüler*in mel- } \\
\text { det sich und } \\
\text { wird aufgerufen }\end{array}$ & $\begin{array}{l}4 \mathrm{a}-\text { Schüler*in mel- } \\
\text { det sich nicht, wird } \\
\text { aber aufgerufen } \\
\text { (Zwangsaufruf) }\end{array}$ & $\begin{array}{l}\text { 4b - Schüler*in wird } \\
\text { nach Beteiligung } \\
\text { erneut aufgerufen } \\
\text { (Rückfrage) }\end{array}$ \\
\hline Ruft nicht auf & $\begin{array}{l}2 \text { - Schüler*in mel- } \\
\text { det sich, } \\
\text { wird aber nicht auf- } \\
\text { gerufen }\end{array}$ & $\begin{array}{l}5 \text { - Schüler*in } \\
\text { meldet sich nicht, } \\
\text { spricht nicht rein und } \\
\text { wird nicht aufgerufen }\end{array}$ & \\
\hline Ruft nicht auf & $\begin{array}{l}3-\text { Schüler*in } \\
\text { spricht unaufge- } \\
\text { fordert rein }\end{array}$ & & \\
\hline
\end{tabular}

Anmerkung. In der Spalte Schüler*in - beteiligt sich nicht werden alle Muster gefasst, welchen keine schüler*innenseitige Initiative zum Anzeigen der Bereitschaft zu einer Unterrichtsbeteiligung vorausgeht

schulische Outcomes entscheidende Indikatoren unterrichtlicher Angebote erwiesen (Klieme et al. 2006; Klieme 2018). Unter der Annahme, dass sich Lehrkräfte hinsichtlich der Qualität ihres Unterrichts unterscheiden (Denn et al. 2019), werden Qualitätsmerkmale primär auf Ebene von Klassen untersucht. Begreift man Lehren und Lernen jedoch als einen interaktiven Prozess (Fend 1998; Helmke 2004), so kann auch das Unterrichtsangebot selbst interindividuell unterschiedlich ausgestaltet sein. Demzufolge kann nicht nur die Nutzung unterrichtlicher Angebote als individuell verschieden betrachtet werden, sondern auch das Unterrichtsangebot selbst variiert interindividuell, beispielweise wenn Lehrkräfte den Lernenden durch selektives Aufrufverhalten unterschiedliche Angebote unterbreiten (Decristan et al. 2019). Entsprechende Annahmen finden sich in Arbeiten zum differential teacher treatment (Bohlmann und Weinstein 2013).

Die Beteiligung am Unterrichtsgespräch stellt, da das Unterrichtsgespräch den umfangreichsten Anteil des Klassenunterrichts ausmacht (Seidel und Prenzel 2006), einen wichtigen Indikator differenzieller Unterrichtsprozesse dar. Dabei kann die Unterrichtsbeteiligung als differenzielles Unterrichtsprozessmerkmal in der ko-konstruktiven Unterrichtsinteraktion betrachtet werden (Vieluf et al. 2020). Diese sichtbaren schüler*innen- und lehrkraftseitigen Unterrichtsaktivitäten führen AngebotsNutzungs-Modellen zufolge wiederum zu inneren Nutzungsprozessen von Schüler*innen. Aktuelle Studien untermauern in diesem Zusammenhang die Bedeutung der Unterrichtsbeteiligung für schulische Outcomes, welche als Ergebnis unterrichtlicher Nutzung gelten (Böheim et al. 2020; Decristan et al. 2019; Ing et al. 2015; Schnitzler et al. 2020).

In Anlehnung an Sacher (1995) lassen sich unterschiedliche Grundmuster der Unterrichtsbeteiligung ausmachen, welche sich wiederum einer schüler*innengesteuerten und einer lehrkraftgesteuerten Beteiligung zuordnen lassen (Decristan et al. 2019; Sacher 1995, vgl. Tab. 1). Von einer schüler*innengesteuerten Beteiligung lässt sich sprechen, ,,wenn eine Beteiligung am Unterricht auf die Initiative der Schülerin bzw. 
des Schülers zurückgeführt werden kann“ (Decristan et al. 2019). Dabei werden sowohl Beteiligungsinitiativen seitens der Schüler*innen, in Form von Meldungen, als auch tatsächliche Wortbeiträge als schüler*innen gesteuerte Beteiligung verstanden (vgl. Tab. 1, linke Spalte „Schüler*in beteiligt sich“). Meldet sich ein*e Schüler*in beispielsweise als Reaktion auf eine Frage der Lehrkraft, einen Gesprächsbeitrag von Mitschüler*innen oder auch gänzlich eigeninitiativ zum Beispiel während einer lehrkraftseitigen Erläuterung, so liegt eine schüler*innengesteuerte Beteiligung vor. Und auch wenn einem Wortbeitrag keine Meldung im Sinne der gewöhnlich geltenden unterrichtlichen Gesprächsregeln (Böheim et al. 2020) voran geht, sondern direkt in den laufenden Unterricht hineingesprochen (Reingerufen) wird, ist dies als schüler*innengesteuerte Beteiligung zu betrachten (Decristan et al. 2019).

Eine lehrkraftgesteuerte Beteiligung liegt dagegen vor, wenn die Auswahl und der Aufruf von Schüler*innen im Unterrichtsgespräch letztendlich auf die Entscheidung einer Lehrkraft zurückgehen (Decristan et al. 2019) (vgl. Tab. 1, obere Zeile „Lehrkraft ruft auf“). Dabei kann eine Lehrkraft das Unterrichtgespräch steuern, indem sie unter mehreren sich meldenden Schüler*innen entscheidet, wer das Rederecht erhalten soll. Alternativ kann eine Lehrkraft im Rahmen eines ,Zwangsaufrufs“" (Lipowsky et al. 2007; Sacher 1995) auch sich nicht eigeninitiativ beteiligende Schüler*innen zur Teilnahme am Unterrichtsgespräch auffordern (Decristan et al. 2019). Das dritte lehrkraftgesteuerte Beteiligungsmuster bildet die Rückfrage einer Lehrkraft an eine*n Schüler*in. Dabei fordert eine Lehrkraft eine*n Schüler*in nach bereits erfolgtem Redeanteil durch eine weitere Frage (Rückfrage) zu einem erneuten Beitrag auf. Eine Rückfrage kann sowohl beruhend auf einem vorangegangenen Aufruf nach Meldung als auch anschließend an einen Zwangsaufruf erfolgen.

Mit Blick auf die zuvor angeführte Unterscheidung zwischen schüler*innengesteuerter und lehrkraftgesteuerter Beteiligung lassen sich entsprechend der Wirkannahmen in Angebots-Nutzungs-Modellen für beide Beteiligungsarten Zusammenhänge mit der individuellen Nutzung von Unterricht erwarten. So konnte Cobb schon 1972 empirisch zeigen (Cobb 1972), dass selbstgesteuert und aktiv am Unterrichtsgeschehen beteiligte Schüler*innen sich mit den gegenwärtigen Unterrichtsinhalten auch mental auseinandersetzen, also on task sind. Angenommen werden kann daher, dass eine schüler*innengesteuerte Beteiligung mit einem höheren Maß an echter Lernzeit (time on task) einhergeht. Zudem lässt sich auf Basis kognitionspsychologischer Ansätze schlussfolgern, dass jede aktive Teilnahme am Unterricht die elaborierte Verarbeitung und verbesserte Integration angeeigneter Wissensinhalte in bestehende mentale Konzepte fördert (Chi und Wylie 2014; Gruber und Stamouli 2020). Darüber hinaus kann eine lehrkraftgesteuerte Beteiligung durch Aufrufen von Schüler*innen und durch Nachfragen Verständnisschwierigkeiten der Lernenden aufgreifen und gezielte Unterstützungsmöglichkeiten bieten (im Sinne eines Scaffoldings; van de Pol et al. 2010; Webb 2009).

Ferner spielt die aktive, schüler*innen- wie auch lehrkraftgesteuerte Unterrichtsbeteiligung für motivationale Prozesse eine wichtige Rolle. Es ist anzunehmen, dass die aktive Beteiligung das Erleben von Selbstbestimmung fördert, indem die Möglichkeit das eigene Wissen im Klassengeschehen zu teilen mit einem gesteigerten Kompetenzerleben einhergeht (Pauli und Lipowsky 2007). Versteht man aktive Beteiligung ergänzend als Indikator für eine Partizipation am ko-konstruktiven Unter- 
Abb. 1 Die Zusammenhänge von individuellen Lernvoraussetzungen, Unterrichtsbeteiligung und Nutzung unterrichtlicher Angebote im Kontext des Angebots-Nutzungs-Modells

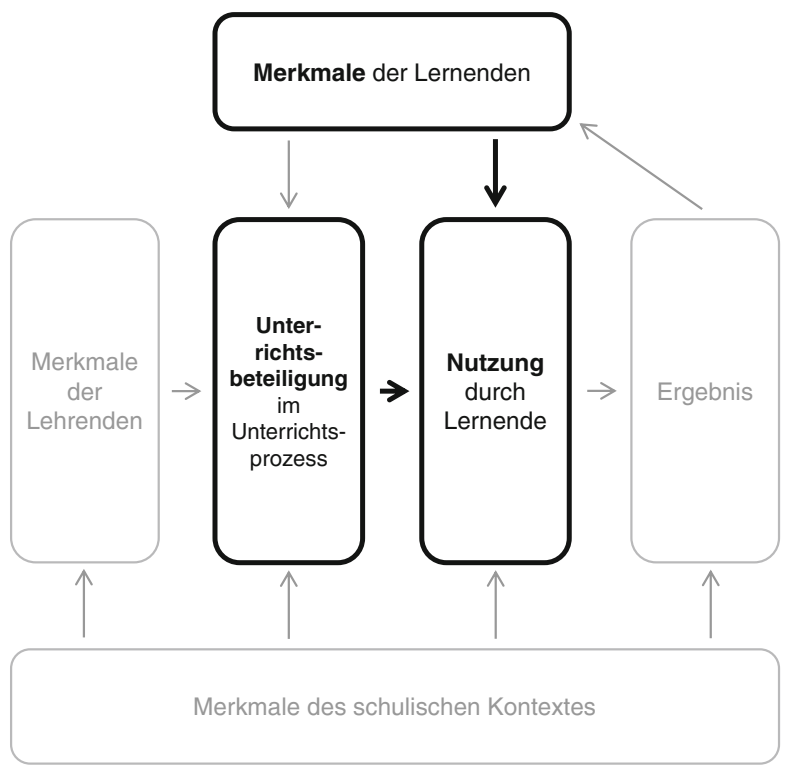

richtsgeschehen (Black 2004; Resnick et al. 2015), sollte eine aktive Unterrichtsbeteiligung überdies auch positiven Einfluss auf das Erleben sozialer Eingebundenheit nehmen (Seidel 2014). Dies ist eine für die lehrkraftgesteuerte Beteiligung zunächst kontraintuitive Annahme, die jedoch durch aktuelle Studien gestützt wird, indem diese die Relevanz von Schüler*innen-Lehrkraft-Interaktionen für das gesamte soziale Gefüge von Lerngruppen hervorheben (Farmer et al. 2011; Schwab und Rossmann 2020). Diese Aspekte ,aktiver und selbstmotivierter, [...] bewusster“ (Reusser 2006, S. 159) Nutzung unterrichtlicher Angebote finden sich auch in der Charakterisierung des umfassenden Konstrukts der kognitiven Aktivierung wieder. Die aktive, schüler*innen- ebenso wie lehrkraftgesteuerte Unterrichtsbeteiligung kann in diesem Zusammenhang als Form des dialogisch ko-konstruierten Wissenserwerbs gelten und als Unterrichtsprozessmerkmal die kognitive Aktivierung effektiv begünstigen (Reusser 2006). Eine Reihe theoretischer (Pianta und Hamre 2009; Praetorius und Charalambous 2018) und empirischer Beiträge (Pauli et al. 2008; Resnick et al. 2015) unterstützen diese Annahme eines grundsätzlichen Zusammenhangs der aktiven Unterrichtsbeteiligung mit selbstberichteter kognitiver Aktivierung von Schüler*innen.

Ein Unterschied in der Bedeutung schüler*innengesteuerter gegenüber lehrkraftgesteuerter Unterrichtsbeteiligung lässt sich für das Autonomieerleben als Facette des Selbstbestimmungserlebens vermuten. Während die aktive, schüler*innengesteuerte Beteiligung als Ausdruck selbstbestimmter Teilhabe das Grundbedürfnis nach Autonomie unterstützen kann (Ruzek et al. 2016; Seidel 2014), erscheinen insbesondere die lehrkraftgesteuerten Zwangsaufrufe geeignet das Autonomieerleben der gegebenenfalls unfreiwillig aufgeforderten Schüler*innen sogar zu untergraben. Jedoch verzeichnen die wenigen Studien, welche lehrkraftgesteuerte Aufrufe ohne schüler*innenseitige Meldung explizit ausweisen, geringe Anteile dieser Beteiligungsform und dementgegen deutlich häufiger lehrkraftgesteuerte Aufrufe 
nach Meldung (Ackermann 2011; Decristan et al. 2019). Infolgedessen erscheint der positive Einfluss schüler*innengesteuerter Beteiligung auf das selbstberichtete Selbstbestimmungserleben der Schüler*innen prominenter, während negative Zusammenhänge mit lehrkraftgesteuerter Beteiligung eine untergeordnete Rolle spielen (Abb. 1).

\section{Fragestellungen und Hypothesen}

Angebots-Nutzungs-Modelle haben sich als Rahmenkonzepte zur Modellierung komplexer unterrichtlicher Prozesse etabliert und wurden in den vergangenen zwei Jahrzehnten vielfältig zur Erklärung unterrichtlicher Wirkungen herangezogen (Kunter und Baumert 2011; Seidel 2014). Bisher lag der Fokus verstärkt auf der Betrachtung des Angebots einerseits, sowie auf der Untersuchung von Leistungsindikatoren als Kriterium andererseits (Böheim et al. 2020; Klieme et al. 2006); unterrichtliche Nutzung selbst wurde bisher kaum empirisch erforscht. Die vorliegende Studie nimmt nun schüler*innenseitige unterrichtliche Nutzungsprozesse näher in den Blick. Als Prädiktoren individueller Nutzung werden individuelle Lernvoraussetzungen und die Unterrichtsbeteiligung als differenzielle Unterrichtsprozesse einbezogen. Zudem soll geprüft werden, ob differenzielle Unterrichtsprozesse über individuelle Lernvoraussetzungen hinaus Unterschiede in der selbstberichteten Nutzung unterrichtlicher Angebote erklären können.

In der vorliegenden Untersuchung werden individuelle Nutzung (kognitive Aktiviertheit, aktive Lernzeit und Selbstbestimmungserleben) und individuelle Lernvoraussetzungen (soziodemographische Merkmale, Vorwissen, Selbstkonzept und Interesse) über Fragebögen und einen Vorwissenstest erfasst. Die individuelle Unterrichtsbeteiligung (schüler*innengesteuerte und lehrkraftgesteuerte Beteiligung) wird hingegen über externe Videokodierungen erfasst.

Der vorliegende Beitrag untersucht folgende Fragestellungen und Hypothesen:

1. Welche Zusammenhänge zeigen sich zwischen individuellen Lernvoraussetzungen und selbstberichteter Nutzung unterrichtlicher Angebote im Mathematikunterricht der Sekundarstufe?

Basierend auf theoretischen Annahmen und empirischen Befunden zur Bedeutung soziodemografischer, kognitiver und motivationaler Merkmale für den Lernerfolg (z.B. Helmke und Weinert 1997) gehen wir davon aus, dass männliches Geschlecht, kein Zuwanderungshintergrund, hoher sozioökonomischer Status sowie Vorwissen, Selbstkonzept und Interesse positiv mit individueller Nutzung des Mathematikunterrichts zusammenhängen.

Mit soziodemographischen Merkmalen verknüpfte, differenzielle eigene Erfolgserwartungen und solche von Lehrkräften (Jussim und Harber 2005) sollten dazu führen, dass Jungen, Schüler*innen ohne Zuwanderungshintergrund und mit hohem sozioökonomischen Status die Lernzeit besser nutzen und Inhalte vertiefter verarbeiten können sowie mehr Selbstbestimmung erleben. Ein höheres Vorwissen sollte zu mehr aktiv genutzter Lernzeit und einer vertieften Auseinandersetzung sowie einer 
verstärkten kognitiven Aktiviertheit durch Anknüpfung an vorhandenen Wissensinhalte führen (Chi und Wylie 2014; Fauth und Leuders 2018; Schraw 2006) sowie mit höherem Selbstbestimmungserleben (vor allem Kompetenzerleben) einhergehen (Großmann et al. 2020). Auch Interesse gilt als grundlegend für das Erleben von Selbstbestimmung (Deci 1992) und sollte zudem aufgrund einer häufigeren und elaborierten Verarbeitung von Inhalten zu mehr aktiver Lernzeit und kognitiver Aktiviertheit führen (Schiefele et al. 1993b). Das fachspezifische Selbstkonzept sollte, vergleichbar mit dem Interesse, zu vermehrter zeitlicher und verstärkter kognitiver Auseinandersetzung mit unterrichtlichen Inhalten (Marsh und Martin 2011) sowie zu mehr Selbstbestimmungserleben führen (Lepper et al. 2021).

2. Welche Zusammenhänge zeigen sich zwischen individueller Unterrichtsbeteiligung und selbstberichteter Nutzung unterrichtlicher Angebote im Mathematikunterricht der Sekundarstufe?

Anknüpfend an die vorangegangenen Erläuterungen werden positive Zusammenhänge zwischen schüler*innengesteuerter und lehrkraftgesteuerter Unterrichtsbeteiligung und individueller Nutzung unterrichtlicher Angebote erwartet. Sowohl für die schüler*innengesteuerte als auch für die lehrkraftgesteuerte Beteiligung werden auf Basis der bisherigen Befundlage positive Zusammenhänge mit allen drei untersuchten Nutzungsparametern, der selbstberichteten aktiven Lernzeit (Chi und Wylie 2014), selbstberichteten kognitiven Aktiviertheit (Pauli et al. 2008) und dem Selbstbestimmungserleben (Kompetenzerleben - Pauli und Lipowsky 2007, Autonomie Ruzek et al. 2016, Eingebundenheitserleben - Seidel 2014) angenommen.

3. Welche Bedeutung hat die individuelle Unterrichtsbeteiligung über die individuellen Lernvoraussetzungen hinaus für die selbstberichtete Nutzung unterrichtlicher Angebote?

Wir erwarten in Anlehnung an Angebots-Nutzungs-Modelle (Seidel 2014; Vieluf et al. 2020), dass die Unterrichtsbeteiligung auch nach Kontrolle der individuellen Lernvoraussetzungen einen eigenständigen Beitrag zur Erklärung der schüler*innenseitigen Nutzung des unterrichtlichen Angebotes leistet.

\section{Methode}

\subsection{Durchführung und Stichprobe}

Analysiert wurden die in Deutschland erhobenen Daten (Grünkorn et al. 2020) der internationalen TALIS-Studie (Teaching and Learning International Survey aus den Jahren 2017/2018, OECD 2020a, b). An der Studie nahmen Lerngruppen der achten bis zehnten Jahrgangsstufe aus insgesamt 38 Schulen (30 Gymnasien, 4 Gesamtschulen, 2 Realschulen, 1 Oberschule, 1 berufsbildende Schule) teil. Vor und nach der Unterrichtsreihe fanden Befragungen und Testungen von Schüler*innen in einem zeitlichen Abstand von durchschnittlich acht Wochen statt. Während der 
Unterrichtsreihe wurden 45- bzw. 90-minütige Unterrichtsstunden zu insgesamt drei Messzeitpunkten videografiert und Sitzpläne mit den individuellen Codes der Lernenden angefertigt. Die Projektteilnahme von Lehrkräften und Lernenden erfolgte freiwillig und unter Einverständnis zur Videografie. Vorgegebenes Thema der videografierten Unterrichtsstunden war die Unterrichtsreihe Quadratische Gleichungen.

Grundlage des vorliegenden Beitrages bilden die Bild- und Tonaufzeichnungen der Klassenkameras zum ersten videografierten Messzeitpunkt. Eine der beiden Kameras erfasste während der gesamten Unterrichtszeit das Klassengeschehen unter Nutzung einer statisch installierten Weitwinkeleinstellung (Klassenkamera). Die andere Kamera verfolgte dynamisch das Handeln der unterrichtenden Lehrkraft (Lehrkraftkamera). Die Tonaufzeichnungen erfolgten durch Raummikrofone an den Kameras.

Unter Anwendung eines time sampling-Verfahrens wurden pro Lerngruppe im Mittel 12,57-minütige Sequenzen $(S D=2,20)$ des klassenöffentlichen Gesprächs selektiert und ausgewertet. Das frontalunterrichtliche Setting des klassenöffentlichen Gesprächs wurde sowohl aufgrund der grundsätzlich für alle Schüler*innen einer Lerngruppe vergleichbaren Chancen zur aktiven Beteiligung als Auswertungsrahmen gewählt als auch bezüglich seiner Repräsentativität hinsichtlich umfangreicher Anteile des Klassenunterrichts (Seidel und Prenzel 2006). In der untersuchten Stichprobe bildete das klassenöffentliche Gespräch, mit Anteilen zwischen $60 \%$ bis $100 \%$ der Unterrichtszeit, im Mittel die häufigste Arbeitsform (Grünkorn et al. 2020). Die zeitliche Eingrenzung der ausgewerteten Abschnitte erfolgte in dem Bestreben, neben der bestehenden thematischen, auch eine größtmögliche strukturelle Vergleichbarkeit zwischen allen inkludierten Lerngruppen zu gewährleisten.

Von den insgesamt 50 in der TALIS-Videostudie aufgezeichneten Lerngruppen wurden diejenigen Klassen ausgeschlossen, in denen entweder kein kontinuierliches, mindestens 10-minütiges Unterrichtsgespräch stattfand oder eine große Anzahl an teilnehmenden Personen infolge ungünstiger Bildausschnitte nicht identifizierbar waren. Demzufolge verringerte sich die Analysestichprobe um zehn Klassen, sodass schließlich 40 Lerngruppen in den vorliegenden Beitrag eingingen. Die Videokodierungen wurden, auf Basis der in Sitzplänen registrierten Schüler*innen-IDs, den Daten von 932 Schüler*innen aus den Fragebogenerhebungen und Tests zugeordnet. Diese waren im Durchschnitt 15 Jahre alt $(S D=0,83)$, 48,9\% gaben eine weibliche Geschlechtszugehörigkeit an und 13,4\% der Teilnehmenden berichteten einen familiären Zuwanderungshintergrund mit mindestens einem nicht in Deutschland geborenen Elternteil. Im Mittel nahmen 23 Schüler*innen pro Lerngruppe an den Erhebungen teil $(S D=3,83$; $\min 16$; $\max 31)$. Für 71 Schüler*innen aus dem Gesamtdatensatz lagen keine videobasierten Beteiligungskodierungen vor, da sie zum Zeitpunkt der Videoaufzeichnungen nicht anwesend oder auf den Aufzeichnungen nicht identifizierbar waren.

\subsection{Instrumente}

Die individuellen Lernvoraussetzungen wurden vor Beginn der Unterrichtsreihe mit Hilfe von durch ein internationales Team entwickelten Skalen (OECD 2020a) erfasst. Das Vorwissen wurde mittels eines im Rahmen der TALIS-Videostudie entwickelten 
Tests mit 30 Aufgaben zu quadratischen Gleichungen erhoben $(\alpha=0,76$; McCaffrey et al. 2020). Das Mathematik-Selbstkonzept wurde mit sechs Items $(\alpha=0,90$; ICC1 =0,04; ICC2 =0,57; Beispielitem: Ich glaube, dass ich anspruchsvolle mathematische Inhalte leicht lernen kann., Mihaly et al. 2021 in Anlehnung an Frey et al. 2009) erfasst. Das fachspezifische Interesse wurde über drei Items einbezogen $(\alpha=0,86$; ICC1 =0,11; ICC2=0,73; Beispielitem: Für Mathematik interessiere ich mich., Mihaly et al. 2021 in Anlehnung an Pekrun et al. 2002; Schiefele et al. 1993b). Die Antworten wurden jeweils auf einer vierstufigen Skala (1= stimme überhaupt nicht $z u$ bis $4=$ stimme völlig $z u$ ) erhoben. Als distaler Parameter individueller Lernvoraussetzungen wurde zudem das objektivierte kulturelle Kapital (Bourdieu 1983/2012) der Familien teilnehmender Schüler*innen als belastbarer Indikator des familiären sozioökonomischen Status erhoben (Bos et al. 2003). Erfasst wurde dazu die Anzahl im häuslichen Umfeld vorhandener Bücher auf einer sechsstufigen Skala ( $1=0-10$ Bücher bis $6=$ mehr als 500 Bücher; vgl. Bos et al. 2012). Vor dem Hintergrund empirischer Befunde, die insbesondere einen sehr geringen familiären sozioökonomischen Status als Risiko für differenzielle Effekte unterrichtlicher Prozesse ausweisen (Atlay et al. 2019), ging die nicht kontinuierlich erfasste Variable in vereinfachter Form dichotomisiert $(0=$ bis zu 100 Bücher, $1=$ mehr als 100 Bücher $)$ in die vorliegenden Analysen ein.

Die Nutzung des Unterrichtsangebots wurde nach Abschluss der Unterrichtsreihe per Fragebogen erfasst. Die Schüler*innen sollten ihre Antworten auf die Unterrichtsreihe beziehen. Als Indikatoren der Nutzung wurden in Anlehnung an das Modell von Klieme et al. (2006) drei im Rahmen der TALIS-Videostudie entwickelte Skalen einbezogen (Mihaly et al. 2021; OECD 2020a): Aktive Lernzeit (3 Items; $\alpha=0,73$; ICC1 = 0,05; Beispielitem: Ich hörte im Unterricht zu.), kognitive Aktiviertheit (3 Items; $\alpha=0,66$; ICC1 =0,04; Beispielitem: Ich dachte intensiv über die mathematischen Themen nach.) und das Selbstbestimmungserleben (3 Items; $\alpha=0,65$; ICC1 =0,11; Autonomie-, Kompetenz- und Eingebundenheitserleben, Beispielitem Autonomieerleben: Ich hatte das Gefühl Dinge selbst entscheiden zu können.). Wie in den vorangehend berichteten Beispielitems erkennbar, erfassten sämtliche betreffenden Itemformulierungen explizit die individuelle Wahrnehmung befragter Schüler*innen in Bezug auf innere Nutzungsaspekte (Ich hörte im Unterricht [...].; Ich dachte intensiv [...]). Damit resultierte eine deutliche sprachliche und inhaltliche Abgrenzung zu Skalen bspw. zur Erfassung des Lehrkrafthandelns oder der Unterrichtsqualität, welche durch explizite Formulierungen wie „Unsere Lehrerin/unser Lehrer [...]“" oder , ,[...] im Unterricht.“ gekennzeichnet sind.

\subsection{Kodierung der Unterrichtsbeteiligung}

Die Kodierung der Unterrichtsbeteiligung erfolgte in einem ersten Schritt über die Einteilung von Fällen als Interaktionseinheiten im klassenöffentlichen Gespräch. Diese wurden definiert als verbale Interaktionen mit inhaltlichem Bezug zur aktuellen Unterrichtsthematik und ferner nach Art der verbalen Beteiligung unterschieden. In die Analysen des vorliegenden Beitrags gingen alle von den Schüler*innen ausgehenden Beteiligungsinitiativen in Form von Reinrufen und Meldungen ein, inklusive Meldungen mit und ohne folgendem verbalem Beitrag (vgl. Beteiligungsmuster 1, 
Tab. 2 Deskriptive Daten der Beteiligungsarten

\begin{tabular}{|c|c|c|c|c|}
\hline & & $\begin{array}{l}\text { Anzahl Fall- } \\
\text { kodierung } \\
n\end{array}$ & $\begin{array}{l}\text { Verteilung Fälle auf } \\
\text { Schüler*innen } \\
n \\
(\% \text { von } 861)\end{array}$ & $M(S D)$ \\
\hline 1 & Aufruf nach Meldung & 581 & $371(43,1)$ & $0,66(0,99)$ \\
\hline 2 & Meldung ohne Aufruf & 3850 & $535(62,1)$ & $4,45(6,38)$ \\
\hline 3 & Reinrufen & 127 & $52(6,0)$ & $0,10(0,53)$ \\
\hline $4 a$ & $\begin{array}{l}\text { Aufruf ohne Meldung } \\
\text { (Zwangsaufruf) }\end{array}$ & 147 & $108(12,5)$ & $0,14(0,42)$ \\
\hline $4 b$ & $\begin{array}{l}\text { Erneuter Aufruf } \\
\text { (Nachfragen) }\end{array}$ & 229 & $173(20,1)$ & $0,23(0,51)$ \\
\hline 5 & - Nicht beteiligt & - & $248(28,8)$ & - \\
\hline \multicolumn{2}{|c|}{ Schüler*innengesteuerte Beteiligung } & 4558 & $364(42,3)$ & $5,21(7,02)$ \\
\hline \multicolumn{2}{|c|}{ Lehrkraftgesteuerte Beteiligung } & 957 & $410(47,6)$ & $1,02(1,44)$ \\
\hline
\end{tabular}

Anmerkung. Schüler*innengesteuerte Beteiligung setzt sich zusammen aus den Beteiligungsarten Aufruf nach Meldung (1), Meldung ohne Aufruf (2) und Reinrufen (3). Lehrkraftgesteuerte Beteiligung setzt sich zusammen aus Aufruf nach Meldung (1) und (erneuter) Aufruf ohne Meldung (4). nicht beteiligt (5) wurde nicht kodiert, sondern anschließend aus allen Schüler*innen ohne Beteiligung in den anderen Mustern bestimmt

2 und 3 in Tab. 1). Als lehrkraftgesteuerte Unterrichtsbeteiligung wurden Aufrufe mit und ohne vorherige Meldung kodiert (vgl. Beteiligungsmuster 1 und $4 \mathrm{a} / \mathrm{b}$ in Tab. 1). Es wurden ausschließlich Beiträge mit inhaltlich-thematischem Bezug zum Mathematikunterricht quantitativ, nach der Häufigkeit ihres Auftretens erfasst.

Die Analysestichprobe der Beteiligung umfasste auf diese Weise 1330 Fälle in 40 Lerngruppen. Zur Berechnung der Urteilsübereinstimmung wurden 12 Klassen (398 Fälle) doppelt kodiert. Hieraus ergab sich eine prozentuale Übereinstimmung von $87 \%$ für die Fallkodierung und $85 \%$ für die kodierte Beteiligungsart, was einem als gut zu bewertenden Cohens Kappa von $\kappa=0,80$ entspricht (Döring und Bortz 2016).

In 172 Fällen (12,9\%) konnte die Art der Beteiligung nicht eindeutig identifiziert werden. Damit verblieben 1158 verwertbare Fälle. In 8 Fällen wurde ein*e Schüler*in nur aufgerufen, um ermahnt zu werden, in 30 Fällen stellte die Lehrkraft Kollektivfragen, die keinem Individuum zugeordnet werden konnten und in 29 Fällen nahmen sich die Schüler*innen gegenseitig dran. Nach Ausschluss dieser Fälle verblieben $N=1091$ Kodierungen. In einem darauffolgenden Schritt wurden alle Meldungen zu den kodierten Fällen über die jeweiligen Schüler*innen-IDs erfasst. Als Meldung definiert wurden dabei Formen des Aufzeigens mit Händen, Armen, aber auch Gegenständen wie Stiften oder Linealen. Alle Meldungen bis zum Beginn einer schüler*innenseitigen Äußerung (Zeitpunkt I) wurden einem Fall zugeordnet (Fall I). Alle nach Beginn dieser schüler*innenseitigen Äußerung und bis zum Beginn einer nächsten schüler*innenseitigen Äußerung (Zeitpunkt II) beobachteten Meldungen wurden dem darauffolgenden Fall (Fall II) zugeordnet. Auf diese Weise wurden $N=3850$ Meldungen erfasst (vgl. Tab. 2). Die Urteilsübereinstimmung lag für diese Kodierungen bei $79 \%$. 
In Tab. 2 ist die Verteilung der Kodierungen auf die unterschiedenen Beteiligungsarten dargestellt.

Mit Hilfe der Schüler*innen-IDs wurden zudem die erfasste Unterrichtbeteiligung mit den Fragebogendaten ( $N=932$ Schüler*innen) zusammengeführt. Da für 71 Schüler*innen aufgrund von Abwesenheit am Videozeitpunkt keine Videokodierungen vorlagen, werden dementsprechend in Tab. 2 die Beteiligungen für 861 Schüler*innen berichtet. Insgesamt war jede Schülerin bzw. jeder Schüler im Mittel 5,63-mal beteiligt $(S D=7,26)$. Etwa ein Viertel der Lernenden war nicht am Unterrichtsgeschehen beteiligt, während für knapp zwei Drittel der Lernenden Meldungen erfasst und über $40 \%$ der Schüler*innen nach Meldung von der Lehrkraft aufgerufen wurden. Sogenannte „Zwangsaufrufe“ (12,5\%) und Reinrufe $(6,0 \%)$ spielten eine vergleichsweise geringe Rolle.

\subsection{Statistische Analysen}

Zur Beantwortung der Hypothesen wurden Mehrebenen-Regressionen eingesetzt. Dabei wurden die individuellen Lernvoraussetzungen und die Unterrichtsbeteiligung auch auf Klassenebene aggregiert. Alle Prädiktor-Variablen wurden sowohl auf der within-Ebene, das heißt hinsichtlich der Zusammenhänge innerhalb von Klassen, als auch auf der between-Ebene, das heißt hinsichtlich der Zusammenhänge zwischen Klassen, in die Analysen einbezogen. Variablen auf der within-Ebene wurden anhand der Gruppenmittelwerte, Variablen auf der between-Ebene anhand des Gesamtmittelwertes zentriert (Enders und Tofighi 2007).

Zur Prüfung der ersten Hypothese wurden die bivariaten Zusammenhänge zwischen den individuellen Lernvoraussetzungen als Prädiktoren und den drei unterrichtlichen Nutzungsindikatoren aktive Lernzeit, kognitive Aktiviertheit und Selbstbestimmungserleben als abhängige Variablen in Mehrebenen-Regressionsmodellen geprüft. Anschließend wurde die spezifische Erklärungskraft der individuellen Lernvoraussetzungen für die Nutzungsindikatoren untersucht, indem alle Merkmale, die signifikant mit den Nutzungsindikatoren zusammenhingen, in ein gemeinsames Regressionsmodell eingingen. Zur Prüfung der zweiten Hypothese wurden schüler*innengesteuerte bzw. lehrkraftgesteuerte Beteiligung jeweils als Prädiktorvariablen für die Nutzungsindikatoren eingesetzt. Für die dritte Fragestellung wurden aus Sparsamkeitsgründen und aufgrund der relativ geringen Stichprobe nur die signifikant mit Nutzung in Beziehung stehenden individuellen Lernvoraussetzungen sowie die jeweiligen Beteiligungsformen simultan als Prädiktorvariablen für die Nutzungsindikatoren in ein gemeinsames Regressionsmodell eingesetzt.

Zum Umgang mit fehlenden Werten (durchschnittlich $8 \%$ pro Variable, max. $10,6 \%$ bei den drei Nutzungsparametern) auf der within-Ebene wurden das FullInformation-Maximum-Likelihood-Verfahren eingesetzt (Arbuckle 1996). Auf der between-Ebene lagen aufgrund der Aggregation der individuellen Werte keine Missings vor. Die Berechnungen erfolgten in MPlus (Muthén und Muthén 2017). Vor dem Hintergrund theoretisch-konzeptioneller Annahmen und der empirischen Befundlage wurde angenommen, dass die untersuchten Variablen positiv zusammenhängen, infolgedessen entfiel die Notwendigkeit einer Betrachtung der Fehlervarianz im ne- 


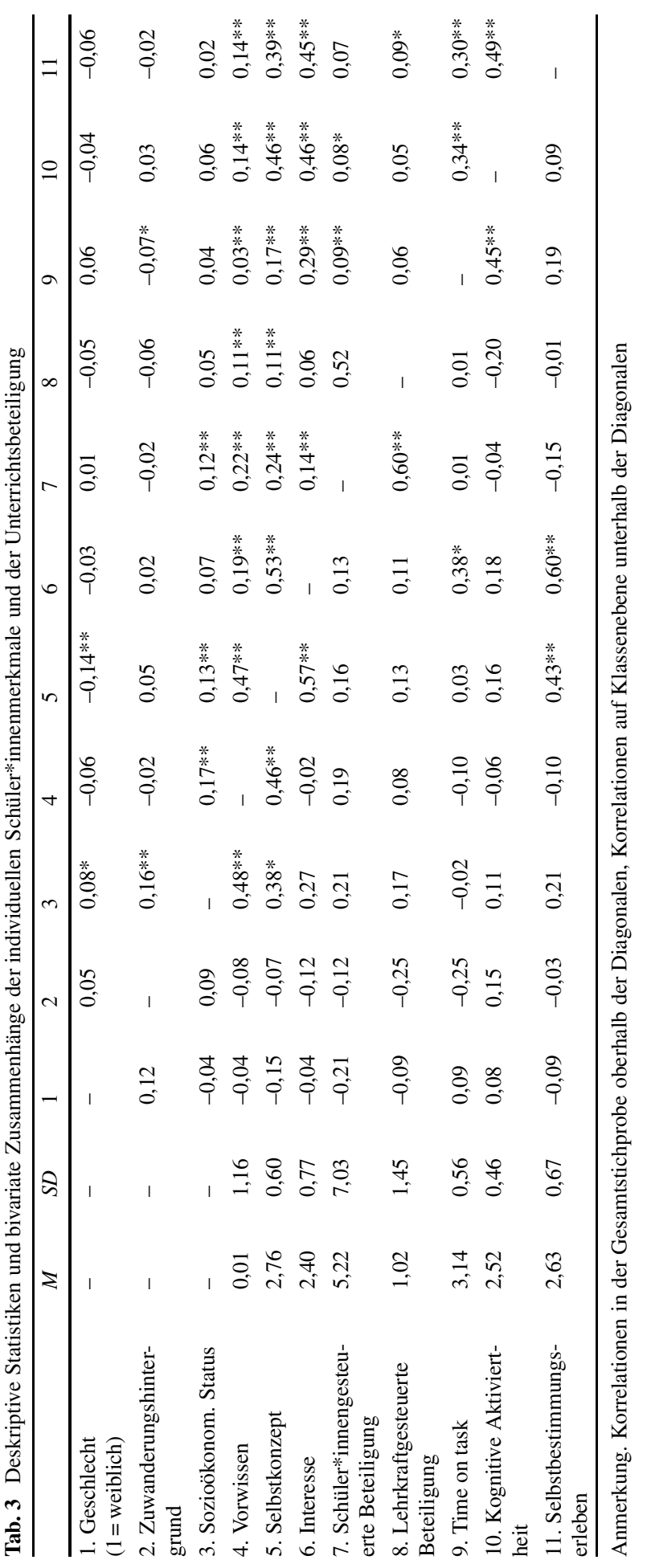


gativen Bereich, sodass eine einseitige Hypothesen-Testung erfolgte (Döring und Bortz 2016).

\section{Ergebnisse}

Die deskriptiven Daten der Fragebogenerhebung und des Vorwissenstests sind in Tab. 3 vermerkt. Hierbei fanden sich insbesondere auf Ebene der Gesamtstichprobe Zusammenhänge zwischen kognitiven und motivationalen Lernvoraussetzungen, Unterrichtsbeteiligung und den drei Nutzungsindikatoren.

\subsection{Individuelle Lernvoraussetzungen und Nutzung}

Zunächst wurde die erste Hypothese von Zusammenhängen individueller Lernvoraussetzungen mit Nutzung geprüft. Die Ergebnisse der Mehrebenen-Regressionsanalysen (s. Tab. 4) zeigten vor allem für die kognitiv-motivationalen Lernvoraussetzungen signifikante Zusammenhänge mit der Nutzung innerhalb von Klassen auf, teils fanden sich entsprechende Zusammenhänge aber auch zwischen Klassen. Für soziodemographische Merkmale zeigten sich wider Erwarten auf der within-Ebene keine Zusammenhänge mit der Nutzung.

Tab. 4 Mehrebenen-Regressionsanalysen zur Vorhersage von Nutzung durch individuelle Merkmale der Lernenden (bivariate Zusammenhänge)

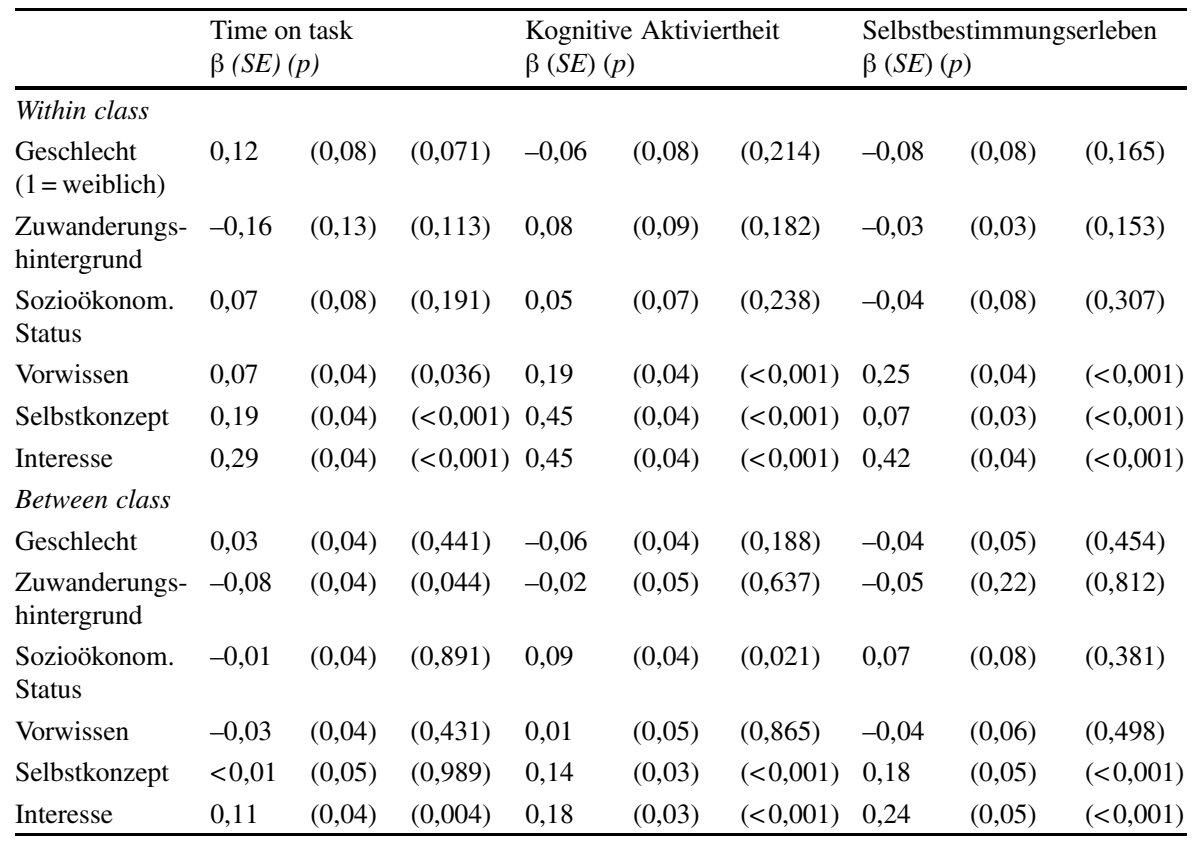

Anmerkung. Jedes individuelle Merkmal wurde einzeln als Prädiktor in die Analysen einbezogen, sodass pro Nutzungsindikator die Ergebnisse sechs einzelner Regressionsanalysen dargestellt sind 
Tab. 5 Mehrebenen-Regressionsanalysen zur Vorhersage von Nutzung durch individuelle Merkmale der Lernenden

\begin{tabular}{|c|c|c|c|c|c|c|c|c|c|}
\hline \multirow[b]{2}{*}{ Within class } & \multicolumn{3}{|c|}{$\begin{array}{l}\text { Time on task } \\
\beta(S E)(p)\end{array}$} & \multicolumn{3}{|c|}{$\begin{array}{l}\text { Kognitive Aktiviertheit } \\
\beta(S E)(p)\end{array}$} & \multicolumn{3}{|c|}{$\begin{array}{l}\text { Selbstbestimmungserleben } \\
\beta(S E)(p)\end{array}$} \\
\hline & & & & & & & & & \\
\hline Vorwissen & $-0,04$ & $(0,05)$ & $(0,250)$ & $-0,07$ & $(0,04)$ & $(0,036)$ & 0,06 & $(0,04)$ & $(0,091)$ \\
\hline Selbstkonzept & 0,08 & $(0,05)$ & $(0,056)$ & 0,34 & $(0,05)$ & $(<0,001)$ & 0,19 & $(0,05)$ & $(<0,001)$ \\
\hline Interesse & 0,25 & $(0,03)$ & $(<0,001)$ & 0,30 & $(0,05)$ & $(<0,001)$ & 0,30 & $(0,04)$ & $(<0,001)$ \\
\hline$\overline{R^{2}}$ & 0,073 & & & 0,258 & & & 0,198 & & \\
\hline \multicolumn{10}{|l|}{ Between class } \\
\hline Vorwissen & 0,03 & $(0,04)$ & $(0,532)$ & 0,01 & $(0,04)$ & $(0,689)$ & $-0,08$ & $(0,06)$ & $(0,138)$ \\
\hline Selbstkonzept & $-0,10$ & $(0,06)$ & $(0,091)$ & 0,04 & $(0,04)$ & $(0,284)$ & 0,11 & $(0,07)$ & $(0,089)$ \\
\hline Interesse & 0,17 & $(0,06)$ & $(0,004)$ & 0,15 & $(0,04)$ & $(<0,001)$ & 0,16 & $(0,06)$ & $(0,010)$ \\
\hline$\overline{R^{2}}$ & 0,351 & & & 0,780 & & & 0,499 & & \\
\hline
\end{tabular}

Tab. 6 Mehrebenen-Regressionsanalysen zur Vorhersage der Nutzung durch die Unterrichtsbeteiligung

\begin{tabular}{|c|c|c|c|c|c|c|c|c|c|}
\hline Within class & \multicolumn{3}{|c|}{$\begin{array}{l}\text { Time on task } \\
\beta(S E)(p)\end{array}$} & \multicolumn{3}{|c|}{$\begin{array}{l}\text { Kognitive Aktiviertheit } \\
\beta(S E)(p)\end{array}$} & \multicolumn{3}{|c|}{$\begin{array}{l}\text { Selbstbestimmungserleben } \\
\beta(S E)(p)\end{array}$} \\
\hline $\begin{array}{l}\text { Schüler*innen- } \\
\text { gesteuerte } \\
\text { Beteiligung }\end{array}$ & 0,14 & $(0,03)$ & $(<0,001)$ & 0,14 & $(0,04)$ & $(<0,001)$ & 0,17 & $(0,04)$ & $(<0,001)$ \\
\hline$R^{2}$ & 0,014 & & & 0,014 & & & 0,023 & & \\
\hline $\begin{array}{l}\text { Lehrkraft- } \\
\text { gesteuerte } \\
\text { Beteiligung }\end{array}$ & 0,07 & $(0,04)$ & $(0,044)$ & 0,06 & $(0,04)$ & $(0,076)$ & 0,13 & $(0,01)$ & $(<0,001)$ \\
\hline$\overline{R^{2}}$ & 0,004 & & & 0,003 & & & 0,016 & & \\
\hline $\begin{array}{l}\text { Between class } \\
\text { Schüler*innen- } \\
\text { gesteuerte } \\
\text { Beteiligung }\end{array}$ & $<0,01$ & $(0,02)$ & $(0,998)$ & $<-0,01$ & $(0,01)$ & $(0,836)$ & $-0,02$ & $(0,01)$ & $(0,089)$ \\
\hline$\overline{R^{2}}$ & $<0,001$ & & & 0,001 & & & 0,033 & & \\
\hline $\begin{array}{l}\text { Lehrkraft- } \\
\text { gesteuerte } \\
\text { Beteiligung }\end{array}$ & $-0,01$ & $(0,09)$ & $(0,959)$ & 0,04 & $(0,07)$ & $(0,565)$ & $-0,01$ & $(0,09)$ & $(0,959)$ \\
\hline$\overline{R^{2}}$ & $<0,001$ & & & 0,012 & & & 0,001 & & \\
\hline
\end{tabular}

Anmerkung. Die Prädiktorvariablen schüler*innengesteuerte bzw. lehrkraftgesteuerte Beteiligung wurden jeweils im Rahmen separater Modelle analysiert

Bei einer simultanen Betrachtung der kognitiven und motivationalen Voraussetzungen (vgl. Tab. 5) stellten das Selbstkonzept und das Interesse weiterhin signifikante Prädiktoren für die Nutzung unterrichtlicher Angebote dar. Das Vorwissen hing bei simultaner Betrachtung der individuellen Merkmale erwartungswidrig nicht oder negativ mit Nutzung zusammen. 
Tab. 7 Mehrebenen-Regressionsanalysen zur Vorhersage der Nutzung durch individuelle Merkmale und schüler*innengesteuerte Unterrichtsbeteiligung

\begin{tabular}{|c|c|c|c|c|c|c|c|c|c|}
\hline Within class & \multicolumn{3}{|c|}{$\begin{array}{l}\text { Time on task } \\
\beta(S E)(p)\end{array}$} & \multicolumn{3}{|c|}{$\begin{array}{l}\text { Kognitive Aktiviertheit } \\
\beta(S E)(p)\end{array}$} & \multicolumn{3}{|c|}{$\begin{array}{l}\text { Selbstbestimmungserleben } \beta \\
(S E)(p)\end{array}$} \\
\hline $\begin{array}{l}\text { Schüler*innen- } \\
\text { gesteuerte } \\
\text { Beteiligung }\end{array}$ & 0,09 & $(0,03)$ & $(<0,001)$ & $<0,01$ & $(0,03)$ & $(0,424)$ & 0,06 & $(0,03)$ & $(0,027)$ \\
\hline Vorwissen & $-0,05$ & $(0,05)$ & $(0,185)$ & $-0,07$ & $(0,04)$ & $(0,040)$ & 0,05 & $(0,05)$ & $(0,131)$ \\
\hline Selbstkonzept & 0,06 & $(0,05)$ & $(0,086)$ & 0,34 & $(0,05)$ & $(<0,001)$ & 0,18 & $(0,04)$ & $(<0,001)$ \\
\hline Interesse & 0,25 & $(0,03)$ & $(<0,001)$ & 0,30 & $(0,05)$ & $(<0,001)$ & 0,30 & $(0,04)$ & $(<0,001)$ \\
\hline$\overline{R^{2}}$ & 0,078 & & & 0,258 & & & 0,200 & & \\
\hline \multicolumn{10}{|l|}{ Between class } \\
\hline $\begin{array}{l}\text { Schüler*innen- } \\
\text { gesteuerte } \\
\text { Beteiligung }\end{array}$ & $<0,01$ & $(0,01)$ & $(0,919)$ & $<-0,01$ & $(0,01)$ & $(0,351)$ & $-0,02$ & $(0,01)$ & $(0,003)$ \\
\hline Vorwissen & 0,03 & $(0,04)$ & $(0,560)$ & 0,02 & $(0,04)$ & $(0,606)$ & $-0,07$ & $(0,05)$ & $(0,175)$ \\
\hline Selbstkonzept & $-0,10$ & $(0,06)$ & $(0,088)$ & 0,05 & $(0,04)$ & $(0,242)$ & 0,12 & $(0,06)$ & $(0,058)$ \\
\hline Interesse & 0,17 & $(0,06)$ & $(0,005)$ & 0,15 & $(0,03)$ & $(<0,001)$ & 0,17 & $(0,06)$ & $(0,002)$ \\
\hline$\overline{R^{2}}$ & 0,348 & & & 0,787 & & & 0,550 & & \\
\hline
\end{tabular}

\subsection{Unterrichtsbeteiligung und Nutzung}

Die Ergebnisse zur Bedeutung von Unterrichtsbeteiligung für die Nutzung von Unterrichtsangeboten (Hypothese 2) sind in Tab. 6 zusammengefasst. Entsprechend theoretischen Annahmen zu differenziellen Unterrichtsangeboten und individuellen Nutzungsprozessen fanden sich Zusammenhänge ausschließlich auf der within-Ebene. Hierbei zeigte sich die schüler*innengesteuerte Beteiligung als prädiktiv für alle drei Nutzungsparameter, während die lehrkraftgesteuerte Beteiligung zwar mit der aktiven Lernzeit und dem Selbstbestimmungserleben, nicht aber mit der kognitiven Aktiviertheit zusammenhing.

\subsection{Individuelle Lernvoraussetzungen, Unterrichtsbeteiligung und Nutzung}

Schließlich wurde angenommen, dass die Unterrichtsbeteiligung auch über individuelle Lernvoraussetzungen hinaus einen Erklärungsbeitrag für die Nutzung leistet (Hypothese 3). Die multiplen Regressionen unter Einbezug sowohl der individuellen Lernvoraussetzungen als auch der individuellen Unterrichtsbeteiligung zeigten einen eigenständigen, kleinen prädiktiven Beitrag der Unterrichtsbeteiligung für die Nutzung unter Kontrolle individueller Lernvoraussetzungen (vgl. Tab. 7 und 8).

Dabei wies die schüler*innengesteuerte Beteiligung auf within-Ebene einen signifikanten Zusammenhang mit der aktiven Lernzeit und dem Selbstbestimmungserleben auf (vgl. Tab. 7). Die lehrkraftgesteuerte Beteiligung verblieb unter Kontrolle individueller Lernvoraussetzungen ausschließlich für das Selbstbestimmungserleben relevant (vgl. Tab. 8). 
Tab. 8 Mehrebenen-Regressionsanalysen zur Vorhersage der Nutzung durch individuelle Merkmale und lehrkraftgesteuerte Unterrichtsbeteiligung

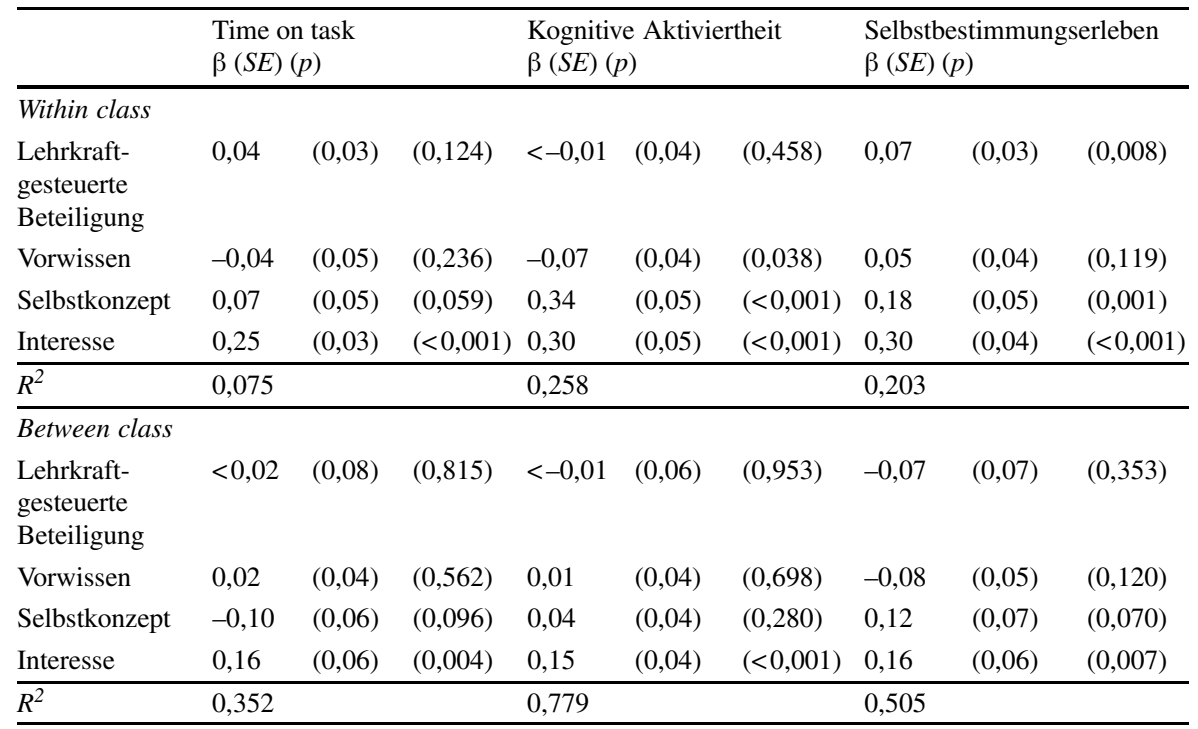

\section{Diskussion}

Die vorliegende Studie konnte bisher wenig untersuchte Zusammenhänge sowohl der kognitiv-motivationalen Merkmale als auch der aktiven Unterrichtsbeteiligung mit der schüler*innenseitigen Nutzung unterrichtlicher Angebote aufzeigen. Dabei legt der vorliegende Beitrag nahe, dass die aktive Unterrichtsbeteiligung einen kleinen, aber eigenständigen Erklärungsbeitrag individueller unterrichtlicher Nutzungsprozesse leisten kann - auch über die Erklärungskraft der individuellen Lernvoraussetzungen hinaus. Der vorliegende Beitrag liefert infolgedessen weitere empirische Hinweise für die Bedeutung differenzieller Unterrichtsprozesse, wie der aktiven Beteiligung, für das schulische Lernen.

Vor dem Hintergrund des Fokus der vorliegenden Untersuchung auf der individuellen Nutzung von Unterrichtsangeboten durch Schüler*innen liegt das Hauptaugenmerk der Interpretation unserer Ergebnisse auf den Unterschieden innerhalb von Lerngruppen (,Analyseebene within“). Infolgedessen werden insbesondere die individuellen Lernvoraussetzungen und differenziellen Unterrichtsprozesse der Schüler*innen im Vergleich zu ihnen Mitschüler*innen betrachtet.

Bezüglich der einzelnen Hypothesen und Befunde zeigten sich die erwarteten $\mathrm{Zu}$ sammenhänge von kognitiv-motivationalen Lernvoraussetzungen mit den von Schüler*innen berichteten Nutzungsindikatoren aktiver Lernzeit, kognitiver Aktiviertheit und dem Erleben von Selbstbestimmung. Die distalen Merkmale standen dagegen wider Erwarten nicht mit der individuellen Nutzung in Zusammenhang. Wenngleich distale im Vergleich zu proximalen Merkmalen eine geringere Bedeutung für das Lernen haben (z.B. Helmke und Weinert 1997), zeigen Studien dennoch systematische Unterschiede in den Unterrichtsinteraktionen abhängig von soziodemo- 
graphischen Merkmalen der Schüler*innen (z.B. Jussim und Harber 2005). Den Zusammenhang von Soziodemographie, Nutzen und Outcome gilt es in zukünftigen Studien weiter zu explorieren. Mit Blick auf proximale Variablen waren das fachspezifische Interesse und das Selbstkonzept für die unterrichtliche Angebotsnutzung durch Schüler*innen besonders relevant. Dieser Befund knüpft an den Forschungsstand zur Bedeutung beider Variablen für selbstgesteuerte Lernhandlungen (Jansen et al. 2019) und schulische Outcomes an. Das Vorwissen stand zwar im bivariaten Zusammenhang mit der Nutzung, leistete unter Kontrolle von Interesse und Selbstkonzept jedoch keinen eigenständigen Erklärungsbeitrag. Vor dem Hintergrund der zentralen Bedeutung des Vorwissens für schulische Outcomes (Helmke und Weinert 1997) gilt es, diesem Befund weiter nachzugehen. So könnte die methodische Nähe bei der Erfassung von Interesse, Selbstkonzept und Nutzung (jeweils erfasst durch Schüler*innen-Fragebögen) diesen Befund mit erklären.

Als zweites wurde die Bedeutung individueller Unterrichtsbeteiligung für die Nutzung untersucht. Zunächst unterstützen die deskriptiven Befunde zur aktiven Unterrichtsbeteiligung die Annahmen des differential teacher treatment (Bohlmann und Weinstein 2013): Die Schüler*innen waren auf unterschiedliche Arten und unterschiedlich häufig am Unterricht beteiligt (s. Tab. 2). Knapp ein Drittel der Teilnehmenden $(28,8 \%)$ war in der ausgewählten Sequenz gar nicht beteiligt und auch eine lehrkraftinitiierte Einbindung durch sogenannte Zwangsaufrufe erfolgte lediglich in $12,5 \%$ aller Kodierungen. Dabei legen frühere Befunde nahe, dass nicht am Unterricht beteiligte Lernende auch geringere fachliche Leistungen zeigen (Decristan et al. 2019), sodass auf diese Lernenden in Zukunft ein verstärktes Augenmerk gelegt werden sollte. Darüber hinaus fanden sich die erwarteten Zusammenhänge zwischen schüler*innengesteuerter Beteiligung mit allen drei Nutzungsparametern. Für die lehrkraftgesteuerte Beteiligung fanden sich signifikante Zusammenhänge dagegen ausschließlich mit der aktiven Lernzeit und dem Selbstbestimmungserleben. Letztgenannter Befund erscheint zunächst kontraintuitiv, verweist er doch auf eine aus Schüler*innenperspektive paradoxe, da scheinbar fremdgesteuerte Bestärkung des Selbstbestimmungserlebens. Die differenzierte Betrachtung der Facetten des Erlebens von Selbstbestimmung, wie es in der vorliegenden Studie erfasst wurde, kann jedoch einen Erklärungsansatz für den vorliegenden Befund bieten. Die lehrkraftgesteuerte Aufforderung kann das Kompetenz- und Autonomieerleben der beteiligten Schüler*innen unterstützen, da Wortbeiträge allgemein geeignet sind, um Selbstwirksamkeitserfahrungen zu bestärken (Pauli und Lipowsky 2007). Und auch für das Eingebundenheitserleben als dritter Facette des Selbstbestimmungserlebens ist die lehrkraftgesteuerte Beteiligung möglicherweise bedeutsam. Aktuelle Forschung zur Relevanz von Lehrkräften für das soziale Gefüge der Schüler*innen innerhalb von Lerngruppen zeigen, dass Interaktionen mit Lehrkräften allgemein, ebenso wie die explizite Beteiligung am Unterricht durch Lehrkräfte, die soziale Position von Schüler*innen im Lerngruppenverband positiv beeinflussen kann (Farmer et al. 2011; Schwab und Rossmann 2020). Das Erleben von Selbstwirksamkeit und Selbstbestimmtheit in Folge von Aufrufen durch die Lehrkraft könnte dementsprechend den positiven Zusammenhang von lehrkraftgesteuerter Beteiligung mit dem Selbstbestimmungserleben mit erklären. Ergänzend trägt möglicherweise auch der erwartbar geringe Anteil von Zwangsaufrufen (Ackermann 2011; Decristan et al. 2019) dazu 
bei, dass der grundsätzlich autonomiefördernde Charakter unterrichtlicher Teilhabe (Seidel 2014) nicht maßgeblich durch Einflüsse potenziell autonomiegefährdender Beteiligungsformen beeinträchtigt wird.

Im Rahmen der dritten Hypothese zur inkrementellen Bedeutung aktiver Beteiligung konnte auch über die kognitiven und motivationalen Lernvoraussetzungen hinaus signifikante Zusammenhänge zwischen Unterrichtsbeteiligung und Nutzung des unterrichtlichen Angebots gefunden werden (vgl. Tab. 7 und 8). Dabei erscheint es bemerkenswert, dass sich zusätzlich zu den deutlichen Zusammenhängen von Selbstkonzept, fachspezifischem Interesse und schüler*innenberichteter Nutzung auch eigenständige Erklärungsbeiträge von schüler*innengesteuerter Beteiligung auf die berichtete aktive Lernzeit und das Selbstbestimmungserleben sowie von lehrkraftgesteuerter Beteiligung auf das Selbstbestimmungserleben fanden. Wenngleich diese Befunde die Relevanz der Unterrichtsbeteiligung für die Nutzungsprozesse von Unterrichtsangeboten unterstreichen, ist der Anteil aufgeklärter Varianz sehr gering.

Hierbei muss jedoch berücksichtigt werden, dass sowohl die motivationalen Lernvoraussetzungen als auch die Nutzungsindikatoren durch ein vergleichbares Instrument (einen Fragebogen) mit gleichem Antwortformat erfasst wurden. Zusammenhänge zwischen beiden sind deshalb vermutlich durch einen common method bias erhöht. Dass die externen Videokodierungen der aktiven Beteiligung dennoch zusätzlich zu den individuellen Lernvoraussetzungen die Nutzung des Unterrichtsangebots erklären konnten, erscheint vor dem Hintergrund unterschiedlicher Datenquellen und dem begrenzten Beobachtungszeitraum somit dennoch bemerkenswert. Die vorliegenden Befunde sollten daher vorsichtig interpretiert, aber dennoch als interessant für die Ausdifferenzierung von Unterrichtsprozessen und deren Zusammenhang mit Nutzung betrachtet werden.

Konzeptionell ist anzumerken, dass Nutzung als interindividuell unterschiedlicher Prozess definiert wurde, woraus Zusammenhänge zwischen individuellen Merkmalen und Unterrichtsbeteiligung auf der within-Ebene fokussiert wurden. Dies erscheint gemeinsam mit den ergänzend angeführten Analysen auf Klassenebene in zweierlei Hinsicht relevant für die Verortung von Beteiligung im unterrichtlichen Gefüge von Angebots- und Nutzungsprozessen. Erstens zeigen die Befunde, dass die Beteiligung aller Lernenden auf Klassenebene keine Bedeutung für die individuelle Nutzung hat und somit nicht unmittelbar auch als Angebot für alle Mitschüler*innen gesehen werden kann (vgl. Diskussion in Vieluf et al. 2020). Vielmehr stellt die Unterrichtsbeteiligung ein interindividuell variierendes Unterrichtsprozessmerkmal dar, welches sich im Sinne des differential teacher treatment (Bohlmann und Weinstein 2013) als differenzielle Angebotsfacette begreifen lässt. Zweitens ist aber auch zu diskutieren, inwiefern vor allem die schüler*innengesteuerte Beteiligung selbst als ein behavioraler Indikator der Nutzung unterrichtlicher Angebote betrachtet werden kann (vgl. Diskussion in Vieluf et al. 2020): Einerseits könnte die quantifizierte Beteiligung selbst als Nutzungsmaß fungieren. Durch die dem vorliegenden Beitrag zugrunde liegende Annahme der Unterrichtsbeteiligung als differenziellem Unterrichtsprozess, sowie vor dem Hintergrund geringer Korrelation zwischen Unterrichtsbeteiligung und selbstberichteter Nutzung, erscheint diese Modellierung jedoch empirisch wenig plausibel. Andererseits wäre auch die Annah- 
me eines Mediationsmodells denkbar, denn Zusammenhänge zwischen individuellen Lernvoraussetzungen und Unterrichtsbeteiligung wurden bereits in mehreren Beiträgen aufgezeigt (Böheim et al. 2020; Decristan et al. 2019; Schnitzler et al. 2020). Aufgrund der konzeptuellen Nähe von Nutzung zu inneren Lernprozessen wurde die Beteiligung in diesem Beitrag jedoch von der Nutzung getrennt betrachtet. Eine weitere Option zur Untersuchung des Zusammenhanges von Unterrichtsbeteiligung und Nutzung stellt die Betrachtung von Unterrichtsbeteiligung als Ausprägung von Nutzungsprozessen dar. Einem entsprechenden Untersuchungsansatz läge die Annahme zugrunde, dass ein günstiges Zusammenwirken individueller Lernvoraussetzungen und innerer, kognitiver Nutzungsprozesse die Voraussetzung für sichtbare Schüler*innenaktivitäten, wie zum Beispiel aktive Beteiligung, bildet. Insbesondere diese äußerlichen Aktivitäten jedoch gelten durch ihren interaktiven Charakter als nicht eindeutig interpretierbar (Vieluf et al. 2020), sodass im vorliegenden Beitrag ein anderer Fokus gesetzt und theoretisch, wie empirisch fundiert wurde (Decristan et al. 2019; Ing et al. 2015; Klieme et al. 2006; Klieme und Rakoczy 2008). Zuletzt erscheint auch eine Modellierung reziproker Einflüsse der Konstrukte denkbar, wie sie Vieluf et al. (2020) in ihrem integrierten Angebots-Nutzungs-Modell bezeichnen. So erscheinen beispielsweise wechselseitige Einflüsse motivationaler Lernvoraussetzungen, interaktiver unterrichtlicher Prozesse und schüler*innenseitiger Nutzung plausibel. Entsprechend komplexe Wirkgefüge konnten jedoch, auf Basis der zur Verfügung stehenden Datenstruktur mit lediglich zwei vergleichbaren Messzeitpunkten, nicht zuverlässig, z.B. durch die Implementation von Pfadmodellen, geprüft werden. Im vorliegenden Beitrag wird daher auf die Untersuchung eines vergleichsweise voraussetzungsarmen Modells fokussiert, welches bereits kleine, jedoch interessante Hinweise geben und somit als Grundlage weiterer empirische Betrachtungen der komplexen, unterrichtlichen Zusammenhänge von Angebot und Nutzung dienen kann.

Zukünftige Untersuchungen könnten darüber hinaus auch mögliche Einflüsse des lehrkraftseitigen Angebots, vor allem der Unterrichtsqualitätsdimensionen, systematisch einbeziehen. Eine Operationalisierung beispielweise durch Unterrichtsqualitätsratings externer Beobachter würde dabei zu einem weiter ausdifferenzierten Verständnis der ko-konstruktiven Prozesse im Unterricht beitragen.

\section{Limitationen}

Die hier vorgestellten Befunde zur individuellen Beteiligung basieren auf ca. 13-minütigen Ausschnitten aus klassenöffentlichen Gesprächen als häufigster Sozialform des Unterrichts und sind somit nur eingeschränkt auf die gesamte Unterrichtsreihe und andere Sozialformen übertragbar. Infolgedessen ist die externe Validität der Befunde eingeschränkt. Zukünftige Forschungsansätze sollten, unter notwendigen methodischen Adaptionen der eingesetzten Instrumente, auch weitere Sozialformen und/oder weitere Zeitfenster oder Messzeitpunkte einbeziehen, um umfassendere Einblicke in unterrichtliche Prozesse und die Stabilität der Einflüsse auf schüler*innenseitige Nutzung zu ermöglichen. Ferner könnte dies auch der weiteren 
empirischen Absicherung der Bedeutung von Unterrichtsbeteiligung für Nutzung und Lernergebnisse dienen.

Hinsichtlich des Selbstbestimmungserlebens fällt die vergleichsweise sparsame Erfassung im Rahmen einer Skala mit nur drei Items ins Auge. Eine detaillierte Erfassung, bspw. durch separate Skalen für die drei Facetten Kompetenz-, Autonomieund Eingebundenheitserleben, hätten möglicherweise differenziertere Einblicke zur individuellen Nutzung von Unterrichtsangeboten ermöglicht.

Die tradierte Erfassung soziodemografischer Merkmale wie Geschlecht oder Migrationshintergrund in einem dichotomen Format begrenzte in der vorliegende Studie auf eine binäre Betrachtung. Zukünftige Studien sollten die Intersektionalität dieser Merkmale bereits bei der Konzeption der Befragungen berücksichtigen.

\section{Zusammenfassung und Implikationen}

Unterrichtliche Nutzung bildet eine wesentliche Säule des schulischen Lernens von Schüler*innen aller Altersgruppen. Die vorliegende Studie liefert nun einen empirischen Beitrag zur Erklärung von Nutzungsprozessen. So konnte einerseits der prädiktive Wert kognitiver und motivationaler Merkmale für die unterrichtliche Nutzung bestätigt werden. Andererseits zeigen die Befunde die Bedeutung aktiver Unterrichtsbeteiligung als zusätzlich relevanten Faktor auf. Somit scheinen die vergleichsweise aufwändigen Videokodierungen der individuellen Beteiligung am Unterrichtsgespräch vielversprechend zur Erklärung von Unterschieden in der individuellen Nutzung des Unterrichtsangebots beizutragen.

Funding Open Access funding enabled and organized by Projekt DEAL.

Open Access Dieser Artikel wird unter der Creative Commons Namensnennung 4.0 International Lizenz veröffentlicht, welche die Nutzung, Vervielfältigung, Bearbeitung, Verbreitung und Wiedergabe in jeglichem Medium und Format erlaubt, sofern Sie den/die ursprünglichen Autor(en) und die Quelle ordnungsgemäß nennen, einen Link zur Creative Commons Lizenz beifügen und angeben, ob Änderungen vorgenommen wurden.

Die in diesem Artikel enthaltenen Bilder und sonstiges Drittmaterial unterliegen ebenfalls der genannten Creative Commons Lizenz, sofern sich aus der Abbildungslegende nichts anderes ergibt. Sofern das betreffende Material nicht unter der genannten Creative Commons Lizenz steht und die betreffende Handlung nicht nach gesetzlichen Vorschriften erlaubt ist, ist für die oben aufgeführten Weiterverwendungen des Materials die Einwilligung des jeweiligen Rechteinhabers einzuholen.

Weitere Details zur Lizenz entnehmen Sie bitte der Lizenzinformation auf http://creativecommons.org/ licenses/by/4.0/deed.de.

\section{Literatur}

Ackermann, S. (2011). Klassengespräch im Mathematikunterricht. Eine Pilotstudie im Rahmen des Projekts „Persönlichkeits- und Lernentwicklung von Grundschulkindern“. Reihe Studium und Forschung, Bd. 19. Kassel: Kassel Univ. Press.

Anderson, L. W. (1976). An empirical investigation of individual differences in time to learn. Journal of Educational Psychology, 68(2), 226-233. 
Arbuckle, J.L. (1996). Full information estimation in the presence of incomplete data. In G. A. Marcoulides \& R. E. Schumacker (Hrsg.), Advanced structural equation modeling (S. 243-277). Mahwah: Lawrence Erlbaum.

Atlay, C., Tieben, N., Fauth, B., \& Hillmert, S. (2019). The role of socioeconomic background and prior achievement for students' perception of teacher support. British Journal of Sociology of Education, 40(7), 970-991. https://doi.org/10.1080/01425692.2019.1642737.

Black, L. (2004). Differential participation in whole-class discussions and the construction of marginalised identities. The Journal of Educational Enquiry, 5(1), 34-54.

Böheim, R., Knogler, M., Kosel, C., \& Seidel, T. (2020). Exploring student hand-raising across two school subjects using mixed methods: an investigation of an everyday classroom behavior from a motivational perspective. Learning and Instruction, 65, 101250. https://doi.org/10.1016/j.learninstruc. 2019.101250.

Bohlmann, N.L., \& Weinstein, R.S. (2013). Classroom context, teacher expectations, and cognitive level: Predicting children's math ability judgments. Journal of Applied Developmental Psychology, 34(6), 288-298. https://doi.org/10.1016/j.appdev.2013.06.003.

Bos, W., Lankes, E.-M., Prenzel, M., Schwippert, K., Walther, G., \& Valtin, R. (2003). Erste Ergebnisse aus IGLU. Schülerleistungen am Ende der vierten Jahrgangsstufe im internationalen Vergleich. Münster: Waxmann.

Bos, W., Wendt, H., Köller, O., \& Selter, C. (Hrsg.). (2012). TIMSS 2011. Mathematische und naturwissenschaftliche Kompetenzen von Grundschulkindern in Deutschland im internationalen Vergleich. Münster: Waxmann.

Bourdieu, P. (2012). Ökonomisches Kapital, kulturelles Kapital, soziales Kapital. In U. Bauer, U.H. Bittlingmayer \& A. Scherr (Hrsg.), Handbuch Bildungs- und Erziehungssoziologie (S. 229-242). Wiesbaden: VS. https://doi.org/10.1007/978-3-531-18944-4_15. (1983/2012).

Carroll, J. B. (1963). A model of school learning. Teacher College Record, 64(8), 723-733.

Chi, M.T.H. (2009). Active-constructive-interactive: a conceptual framework for differentiating learning activities. Topics in Cognitive Science, 1(1), 73-105. https://doi.org/10.1111/j.1756-8765.2008. 01005.x.

Chi, M.T.H., \& Wylie, R. (2014). The ICAP framework: linking cognitive engagement to active learning outcomes. Educational Psychologist, 49(4), 219-243. https://doi.org/10.1080/00461520.2014. 965823.

Cobb, J.A. (1972). Relationship of discrete classroom behaviors to fourth-grade academic achievement. Journal of Educational Psychology, 63(1), 74-80. https://doi.org/10.1037/h0032247.

Craik, F.I., \& Lockhart, R.S. (1972). Levels of processing: A framework for memory research. Journal of Verbal Learning and Verbal Behavior, 11(6), 671-684. https://doi.org/10.1016/S00225371(72)80001-X.

Deci, E.L. (1992). The relation of interest to the motivation of behavior: A self-determination theory perspektive. In K. A. Renninger, S. Hidi \& A. Krapp (Hrsg.), The role of interest in learning and development (S. 43-47). Hillsdale, New Jersey: Erlbaum.

Deci, E.L., \& Ryan, R. M. (1993). Die Selbstbestimmungstheorie der Motivation und ihre Bedeutung für die Pädagogik. Zeitschrift für Pädagogik, 39(2), 223-238.

Decristan, J., Fauth, B., Heide, E. L., Locher, F. M., Troll, B., Kurucz, C., et al. (2019). Individuelle Beteiligung am Unterrichtsgespräch in Grundschulklassen: Wer ist (nicht) beteiligt und welche Konsequenzen hat das für den Lernerfolg? Zeitschrift für Pädagogische Psychologie, 34(3-4), 171-186. https:// doi.org/10.1024/1010-0652/a000251.

Denn, A.-K., Gabriel-Busse, K., \& Lipowsky, F. (2019). Unterrichtsqualität und Schülerbeteiligung im Mathematikunterricht des zweiten Schuljahres. In K. Verrière \& L. Schäfer (Hrsg.), Interaktion im Klassenzimmer (S. 9-29). Wiesbaden: Springer. https://doi.org/10.1007/978-3-658-23173-6_2.

Döring, N., \& Bortz, J. (2016). Forschungsmethoden und Evaluation in den Sozial- und Humanwissenschaften. Berlin, Heidelberg: Springer. https://doi.org/10.1007/978-3-642-41089-5.

Enders, C., \& Tofighi, D. (2007). Centering predictor variables in cross-sectional multilevel models: A new look at an old issue. Psychological Methods, 12, 121-138.

Farmer, T.W., McAuliffe Lines, M., \& Hamm, J. V. (2011). Revealing the invisible hand: The role of teachers in children's peer experiences. Journal of Applied Developmental Psychology, 32(5), 247-256. https://doi.org/10.1016/j.appdev.2011.04.006.

Fauth, B., \& Leuders, T. (2018). Kognitive Aktivierung im Unterricht. Stuttgart: Landesinstitut für Schulentwicklung. 
Fauth, B., Decristan, J., Rieser, S., Klieme, E., \& Büttner, G. (2014). Student ratings of teaching quality in primary school: Dimensions and prediction of student outcomes. Learning and Instruction, 29, 1-9. https://doi.org/10.1016/j.learninstruc.2013.07.001.

Fend, H. (1998). Qualität im Bildungswesen: Schulforschung zu Systembedingungen, Schulprofilen und Lehrerleistung. Weinheim: Juventa.

Frey, A., Taskinen, P., Schütte, K., Prenzel, M., Artelt, C., Baumert, J., et al. (2009). PISA 2006 Skalenhandbuch. Dokumentation der Erhebungsinstrumente. Münster: Waxmann.

Friedrich, H.F., \& Mandl, H. (Hrsg.). (1992). Lern- und Denkstrategien : Analyse und Intervention. Göttingen: Hogrefe.

Gettinger, M. (1984). Individual differences in time needed for learning: A review of literature. Educational Psychologist, 19(1), 15-29. https://doi.org/10.1080/00461528409529278.

Großmann, N., Eckes, A., \& Wilde, M. (2020). Prädiktoren der Kompetenzwahrnehmung von Schülerinnen und Schülern im Biologieunterricht. Zeitschrift für Pädagogische Psychologie. https://doi.org/10. 1024/1010-0652/a000276.

Gruber, H., \& Stamouli, E. (2020). Intelligenz und Vorwissen. In E. Wild \& J. Möller (Hrsg.), Pädagogische Psychologie. Berlin, Heidelberg: Springer.

Grünkorn, J., Klieme, E., Praetorius, A.-K., \& Schreyer, P. (Hrsg.). (2020). Mathematikunterricht im internationalen Vergleich. Ergebnisse aus der TALIS-Videostudie Deutschland. Frankfurt a.M.: DIPF I Leibniz-Institut für Bildungsforschung und Bildungsinformation.

Hattie, J. (2009). Visible learning: A synthesis of over 800 meta analyses relating to achievement. London: Routledge.

Helmke, A. (2004). Unterrichtsqualität erfassen, bewerten, verbessern (3. Aufl.). Seelze: Kallmeyer.

Helmke, A. (2017). Unterrichtsqualität und Lehrerprofessionalität. Diagnose Evaluation und Verbesserung des Unterrichts (7. Aufl.). Seelze: Kallmeyer.

Helmke, A., \& Weinert, F.E. (1997). Bedingungsfaktoren schulischer Leistungen. In F. E. Weinert (Hrsg.), Psychologie des Unterrichts und der Schule (Bd. 3, S. 71-176). Göttingen: Hogrefe.

Ing, M., Webb, N. M., Franke, M.L., Turrou, A.C., Wong, J., Shin, N., et al. (2015). Student participation in elementary mathematics classrooms: the missing link between teacher practices and student achievement? Educational Studies in Mathematics, 90(3), 341-356. https://doi.org/10.1007/s10649-0159625-z.

Jansen, M., Schneider, R., Schipolowski, S., \& Henschel, S. (2019). Motivationale Schülermerkmale im Fach Mathematik und in den naturwissenschaftlichen Fächern. In P. Stanat, S. Schipolowski, N. Klein \& S. Weirich (Hrsg.), IQB-Bildungstrend 2018. Mathematische und naturwissenschaftliche Kompetenzen am Ende der Sekundarstufe I im zweiten Ländervergleich (S. 337-354).

Jussim, L., \& Harber, K.D. (2005). Teacher expectations and self-fulfilling prophecies: knowns and unknowns, resolved and unresolved controversies. Personality and Social Psychology Review, 9, 131-155. https://doi.org/10.1207/s15327957pspr0902_3.

Klauer, K.J., \& Leutner, D. (2012). Lehren und Lernen: Einführung in die Instruktionspsychologie (2. Aufl.). Weinheim: Beltz.

Klieme, E. (2018). Unterrichtsqualität. In M. Haring, C. Rohlfs \& M. Gläser-Zikuda (Hrsg.), Handbuch Schulpädagogik (S. 393-398). Bad Heilbrunn: UTB.

Klieme, E., \& Rakoczy, K. (2008). Empirische Unterrichtsforschung und Fachdidaktik. Outcome-orientierte Messung und Prozessqualität des Unterrichts. Zeitschrift für Pädagogik, 54(2), 222-237.

Klieme, E., Lipowsky, F., Rakoczy, K., \& Ratzka, N. (2006). Qualitätsdimensionen und Wirksamkeit von Mathematikunterricht. Theoretische Grundlagen und ausgewählte Ergebnisse des Projekts „Pythagoras“. In M. Prenzel \& L. Allolio-Näcke (Hrsg.), Untersuchungen zur Bildungsqualität von Schule. Abschlussbericht des DFG-Schwerpunktprogramms (S. 127-146). Münster: Waxmann.

Kriegbaum, K., Becker, N., \& Spinath, B. (2018). The relative importance of intelligence and motivation as predictors of school achievement: A meta-analysis. Educational Research Review, 25, 120-148. https://doi.org/10.1016/j.edurev.2018.10.001.

Kunter, M., \& Baumert, J. (Hrsg.). (2011). Professionelle Kompetenz von Lehrkräften. Ergebnisse des Forschungsprogramms COACTIV

Kunter, M., \& Trautwein, U. (2013). Psychologie des Unterrichts. Paderborn: Schöningh.

Lepper, C., Stang, J., \& McElvany, N. (2021). Bedeutung der wahrgenommenen Motivierungsqualität für intrinsische Motivation und Selbstkonzept von Grundschulkindern. Unterrichtswissenschaft. https:// doi.org/10.1007/s42010-021-00121-y.

Lipowsky, F., Rakoczy, K., Pauli, C., Reusser, K., \& Klieme, E. (2007). Gleicher Unterricht - gleiche Chancen für alle? Die Verteilung von Schülerbeiträgen im Klassenunterricht. Unterrichtswissenschaft, 35(2), 125-147. 
Marsh, H.W., \& Martin, A.J. (2011). Academic self-concept and academic achievement: relations and causal ordering. The British Journal of Educational Psychology, 81(Pt 1), 59-77. https://doi.org/10. 1348/000709910X503501.

McCaffrey, D.F., Castellano, K.E., \& van Essen, T. (2020). Student test development. In OECD (Hrsg.), Global teaching insights technical report. Paris: OECD Publishing.

Mercer, N., \& Dawes, L. (2014). The study of talk between teachers and students, from the 1970s until the 2010s. Oxford Review of Education, 40(4), 430-445. https://doi.org/10.1080/03054985.2014.934087.

Mihaly, K., Klieme, E., Fischer, J. \& Doan, S. (2021). Questionnaire Scale Characteristics. In OECD (Ed.), Global Teaching InSights Technical Report. Paris: OECD Publishing.

Muthén, L. K., \& Muthén, B. O. (2017). MPlus (Version 8)

OECD (2020a). Global Teaching Insights: A Video Study of Teaching. Paris: OECD Publishing.

OECD (2020b). Global Teaching Insights: A Video Study of Teaching. Primärdaten. Verfügbar unter: https://www.oecd.org/education/school/global-teaching-insights-technical-documents.htm.

Pauli, C., \& Lipowsky, F. (2007). Mitmachen oder zuhören? Mündliche Schülerinnen- und Schülerbeteiligung im Mathematikunterricht. Unterrichtswissenschaft, 35(2), 101-124.

Pauli, C., Drollinger-Vetter, B., Hugener, I., \& Lipowsky, F. (2008). Kognitive Aktivierung im Mathematikunterricht. Zeitschrift für Pädagogische Psychologie, 22(2), 127-133. https://doi.org/10.1024/ 1010-0652.22.2.127.

Pekrun, R., Goetz, T., Jullien, S., Zirngibl, A., Hofe, R., \& Blum, W. (2002). Skalenhandbuch Palma 1. Messzeitpunkt (5. Jahrgangsstufe). München: Universität München.

Pianta, R.C., \& Hamre, B.K. (2009). Conceptualization, measurement, and improvement of classroom processes: standardized observation can leverage capacity. Educational Researcher, 38(2), 109-119. https://doi.org/10.3102/0013189X09332374.

van de Pol, J., Volman, M., \& Beishuizen, J. (2010). Scarffolding in teacher-student interaction: a decade of research. Educational Psychology Review, 22(3), 271-296. https://doi.org/10.1007/s10648-0109127-6.

Praetorius, A.-K., \& Charalambous, C. Y. (2018). Classroom observation frameworks for studying instructional quality: looking back and looking forward. ZDM, 50(3), 535-553. https://doi.org/10.1007/ s11858-018-0946-0.

Rakoczy, K. (2006). Motivationsunterstützung im Mathematikunterricht. Zur Bedeutung von Unterrichtsmerkmalen für die Wahrnehmung von Schülerinnen und Schüler. Zeitschrift für Pädagogik, 52(6), 822-843.

Resnick, L., Asterhan, C., \& Clarke, S. (2015). Socializing intelligence through academic talk and dialogue. Washington: American Educational Research Association.

Reusser, K. (2006). Konstruktivismus - vom epistemologischen Leitbegriff zur Erneuerung der didaktischen Kultur. In M. Baer, K. Reusser, M. Fuchs, P. Füglister \& H. Wyss (Hrsg.), Didaktik auf psychologischer Grundlage. Von Hans Aeblis kognitionspsychologischer Didaktik zur modernen Lehr- und Lernforschung (1. Aufl. S. 151-168). Bern: hep.

Ruzek, E. A., Hafen, C.A., Allen, J.P., Gregory, A., Mikami, A. Y., \& Pianta, R. C. (2016). How teacher emotional support motivates students: The mediating roles of perceived peer relatedness, autonomy support, and competence. Learning and Instruction, 42, 95-103. https://doi.org/10.1016/j. learninstruc.2016.01.004.

Sacher, W. (1995). Meldungen und Aufrufe im Unterrichtsgespräch. Theoretische Grundlagen Forschungsergebnisse Trainingselemente und Diagnoseverfahren. Augsburg: Wißner.

Schiefele, U., Krapp, A., \& Schreyer, I. (1993b). Metaanalyse des Zusammenhangs von Interesse und schulischer Leistung. Zeitschrift für Entwicklungspsychologie und Pädagogische Psychologie, 25(2), 120.

Schiefele, U., Krapp, A., Wild, K.P., \& Winteler, A. (1993a). Der „Fragebogen zum Studieninteresse“ (FSI). Diagnostica, 39(4), 335-351.

Schnitzler, K., Holzberger, D., \& Seidel, T. (2020). All better than being disengaged: Student engagement patterns and their relations to academic self-concept and achievement. European Journal of Psychology of Education. https://doi.org/10.1007/s10212-020-00500-6.

Schraw, G. (2006). Knowledge: Structures and processes. In P. A. Alexander \& P. H. Winne (Hrsg.), Handbook of educational psychology (2. Aufl. S. 245-263). New York: Routledge, Taylor \& Francis.

Schwab, S., \& Rossmann, P. (2020). Peer integration, teacher-student relationships and the associations with depressive symptoms in secondary school students with and without special needs. Educational Studies, 46(3), 302-315. https://doi.org/10.1080/03055698.2019.1584852.

Seidel, T. (2014). Angebots-Nutzungs-Modelle in der Unterrichtspsychologie. Integration von Strukturund Prozessparadigma. Zeitschrift für Pädagogik, 60(6), 850-866. 
Seidel, T., \& Prenzel, M. (2006). Stability of teaching patterns in physics instruction: Findings from a video study. Learning and Instruction, 16(3), 228-240. https://doi.org/10.1016/j.learninstruc.2006.03.002.

Taylor, G., Jungert, T., Mageau, G. A., Schattke, K., Dedic, H., Rosenfield, S., et al. (2014). A self-determination theory approach to predicting school achievement over time: the unique role of intrinsic motivation. Contemporary educational psychology, 39(4), 342-358. https://doi.org/10.1016/j.cedpsych. 2014.08.002.

Vieluf, S., Praetorius, A.-K., Rakoczy, K., Kleinknecht, M., \& Pietsch, M. (2020). Angebots-NutzungsModelle der Wirkweise des Unterrichts. In A.-K. Praetorius, J. Grünkorn \& E. Klieme (Hrsg.), Empirische Forschung zu Unterrichtsqualität. Theoretische Grundfragen und quantitative Modellierungen 1. Aufl. Zeitschrift für Pädagogik, Beiheft April 2020, (Bd. 66, S. 63-80).

Vygotskij, L.S. (1978). Mind in society. The development of higher psychological processes. Cambridge: Harvard University Press.

Webb, N. M. (2009). The teacher's role in promoting collaborative dialogue in the classroom. The British Journal of Educational Psychology, 79(1), 1-28. https://doi.org/10.1348/000709908X380772.

Wigfield, A., \& Eccles, J. (2000). Expectancy-value theory of achievement motivation. Contemporary Educational Psychology, 25(1), 68-81. https://doi.org/10.1006/ceps.1999.1015. 